\title{
Nuclear Singlet Relaxation by Chemical Exchange
}

Christian Bengs, ${ }^{1}$ Laurynas Dagys, ${ }^{1}$ Gamal A. I. Moustafa, ${ }^{1}$ James W. Whipham, ${ }^{1}$ Mohamed Sabba, ${ }^{1}$ Alexey S.

Kiryutin, ${ }^{2}$ Konstantin L. Ivanov, ${ }^{2}$ and Malcolm H. Levitt ${ }^{1, a)}$

1) School of Chemistry, Southampton

${ }^{2)}$ International Tomography Center SB RAS, Novosibirsk, Russia

(Dated: September 2, 2021)

The population imbalance between nuclear singlet states and triplet states of strongly coupled spin-1/2 pairs, also known as nuclear singlet order, is well protected against several common relaxation mechanisms. We study the nuclear singlet relaxation of ${ }^{13} \mathrm{C}$ pairs in aqueous solutions of $1,2-{ }^{13} \mathrm{C}_{2}$ squarate, over a range of $\mathrm{pH}$ values. The ${ }^{13} \mathrm{C}$ singlet order is accessed by introducing ${ }^{18} \mathrm{O}$ nuclei in order to break the chemical equivalence. The squarate dianion is in chemical equilibrium with hydrogen-squarate $\left(\mathrm{SqH}^{-}\right)$and squaric acid $\left(\mathrm{SqH}_{2}\right)$ characterised by the dissociation constants $\mathrm{pK}_{1}=1.5$ and $\mathrm{pK}_{2}=3.4$. Surprisingly, we observe a striking increase in the singlet decay time constants $T_{S}$ when the $\mathrm{pH}$ of the solution exceeds $\sim 10$, which is far above the acid-base equilibrium points. We derive general rate expressions for chemical-exchange-induced nuclear singlet relaxation and provide a qualitative explanation of the $T_{S}$ behaviour of the squarate dianion. We identify a kinetic contribution to the singlet relaxation rate constant which depends explicitly on kinetic rate constants. Qualitative agreement is achieved between the theory and the experimental data. This study shows that infrequent chemical events may have a strong effect on the relaxation of nuclear singlet order.

\section{INTRODUCTION}

Long-lived nuclear spin states (LLS) are configurations of nuclear spins which are protected against common relaxation or dissipation mechanisms and which display extended decay time constants ${ }^{1-19}$. A seminal example is the singlet order of a spin- $1 / 2$ pair ensemble, corresponding to a population imbalance between the singlet and triplet states of the spin- $1 / 2$ pairs $^{10,16}$. The decay time constants of such states are often many times the spinlattice relaxation time constant $T_{1}$, with lifetimes exceeding 1 hour being observed in favourable cases ${ }^{12,19}$. Longlived states have been applied to the study of slow chemical and transport processes ${ }^{20-29}$, to the characterisation of biomolecular ligand binding ${ }^{30-34}$, and for the transport and storage of nuclear hyperpolarization ${ }^{5,7,35-43}$. Longlived nuclear singlet order plays a central role in the generation of nuclear hyperpolarization from hydrogen gas enriched in the para spin isomer ${ }^{44-59}$.

The relaxation mechanisms of LLS have been extensively studied ${ }^{3,6,15,60-71}$. Most attention has been given to the dipole-dipole (DD), chemical shift anisotropy (CSA) and random field relaxation mechanisms ${ }^{3,6,60,61,64,68}$. More unusual relaxation mechanisms such as nuclear spin-rotation and scalar relaxation of the second kind have also been identified ${ }^{4,15,65-67,69}$. Generally speaking, the efficiency of a relaxation mechanism is roughly proportional to the rotational correlation time $\tau_{c}$ for the random tumbling of the molecules in solution. This suggests that small molecules, which tumble more rapidly in solution and have small values of $\tau_{c}$, tend to have the longest LLS lifetimes. In most cases, that is also the observed behaviour.

\footnotetext{
a)mhl@soton.ac.uk
}

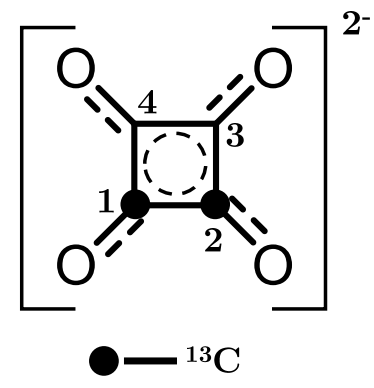

Figure 1. The $1,2-{ }^{13} \mathrm{C}_{2}$-squarate dianion. In the absence of ${ }^{13} \mathrm{C}$ labelling, the ion belongs to the point group $D_{4 h}$ and has 4 -fold rotational symmetry. In the current work, random ${ }^{18} \mathrm{O}$ enrichment is used to slightly break the chemical equivalence of the ${ }^{13} \mathrm{C}$ sites by means of secondary isotope shifts.

When labelled with two ${ }^{13} \mathrm{C}$ nuclei, the small, highly symmetrical squarate molecule is an attractive candidate for exhibiting long-lived ${ }^{13} \mathrm{C}_{2}$ singlet order in aqueous solution (see figure 1). The squarate dianion is stabilised by electron delocalisation and exhibits four-fold rotational symmetry ${ }^{72-75}$. Since the dominant oxygen isotope ${ }^{16} \mathrm{O}$ has no nuclear spin, the ${ }^{13} \mathrm{C}$ pair of $1,2-$ ${ }^{13} \mathrm{C}_{2}$-squarate is magnetically isolated, eliminating all intramolecular dipole-dipole contributions to singlet relaxation. Furthermore, it is technically feasible to introduce non-magnetic ${ }^{18} \mathrm{O}$ nuclei into the squarate dianion, as has been achieved previously for oxalate ${ }^{76}$. The increased mass of the ${ }^{18} \mathrm{O}$ nuclei modifies the vibrational wavefunctions, inducing small secondary isotope shifts of the ${ }^{13} \mathrm{C}$ resonances ${ }^{77}$. These shifts may break the exact magnetic equivalence of the ${ }^{13} \mathrm{C}$ nuclei, providing access to long-lived ${ }^{13} \mathrm{C}_{2}$ singlet order. Large values of the singlet relaxation time constant $T_{S}$ are therefore anticipated for the ${ }^{13} \mathrm{C}$ pair in ${ }^{18} \mathrm{O}$-enriched $1,2-{ }^{13} \mathrm{C}_{2}$-squarate, especially at low magnetic field, when the chemical shift 


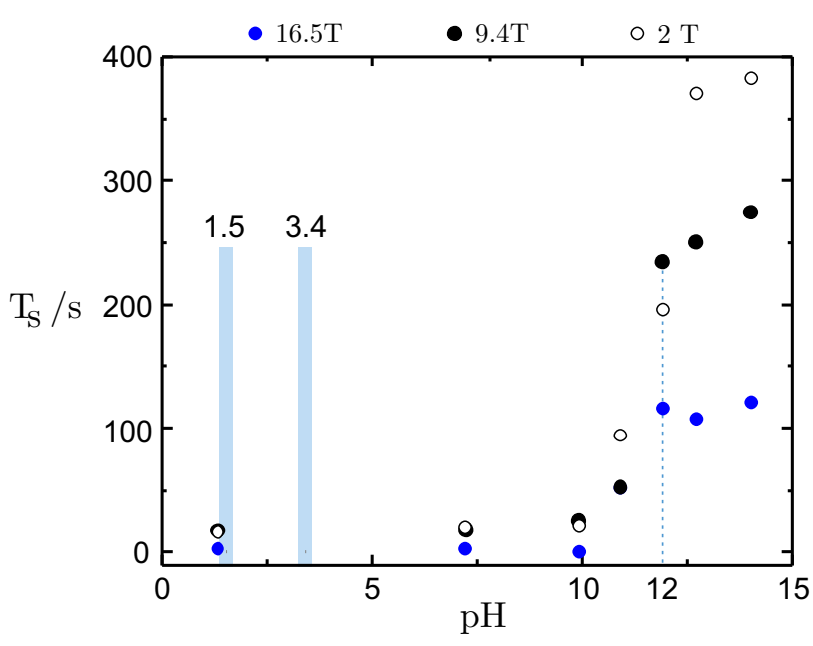

Figure 2. Singlet decay time constants for the ${ }^{13} \mathrm{C}_{2}$ pairs of ${ }^{18} \mathrm{O}$-enriched $1,2-{ }^{13} \mathrm{C}_{2}$ squaric acid in $\mathrm{H}_{2}{ }^{18} \mathrm{O}: \mathrm{D}_{2}{ }^{16} \mathrm{O}$ solution as a function of $\mathrm{pH}$ at three different magnetic fields. The blue bars indicate the $\mathrm{pK}_{\mathrm{a}}$ values for the chemical equilibria between squaric acid $\rightleftharpoons$ hydrogen-squarate and hydrogensquarate $\rightleftharpoons$ sqarate.

anisotropy mechanism of singlet relaxation is removed.

As described below, our initial measurements of the singlet decay time constant $T_{S}$ for the ${ }^{13} \mathrm{C}_{2}$ pairs in ${ }^{18} \mathrm{O}$-enriched $1,2-{ }^{13} \mathrm{C}_{2}$ squarate were disappointing. In aqueous solution and neutral $\mathrm{pH}$ we observed a singlet decay time constant of around $T_{S} \sim 30 \mathrm{~s}$ in a field of $9.4 \mathrm{~T}$, which is slightly shorter than the value of $T_{1}$ under the same conditions. Conventional mechanisms such as CSA-induced relaxation are insufficient to explain this low value of $T_{S}$.

Furthermore, the observed value of $T_{S}$ for ${ }^{18} \mathrm{O}$-enriched $1,2-{ }^{13} \mathrm{C}_{2}$ squarate was found to be strongly dependent on $\mathrm{pH}$, with a rather sharp transition from short $T_{S}$ values at low $\mathrm{pH}$ to long $T_{S}$ values at high $\mathrm{pH}$, approaching $300 \mathrm{~s}$ in low magnetic field (see figure 2).

The strong $\mathrm{pH}$-dependence of $T_{S}$ suggests that the reversible protonation of squarate in aqueous solution plays a role in the singlet relaxation. In aqueous solution, the symmetrical squarate dianion is in equilibrium with hydrogen squarate and squaric acid ${ }^{78}$ (see figure 3 ). The possibility arises that this dynamic chemical process could act as a relaxation mechanism for nuclear singlet order. However, at first sight, this appears unlikely. The $\mathrm{pK}_{\mathrm{a}}$ values for the two deprotonation stages of squaric acid are 1.5 and 3.4 respectively ${ }^{79}$. The equilibrium fractions of the monoprotonated and diprotonated species at a pH of 7.0 are only $\sim 250 \mathrm{ppm}$ and $\sim 0.8 \mathrm{ppb}$ respectively. Such minority species could only have a significant effect if a mechanism exists that leads to an extreme sensitivity of nuclear singlet order to rare protonation events.

A chemical exchange mechanism for singlet relaxation has been proposed before, in the context of ligand binding $^{30-34}$. Those reports assume that the singlet relaxation rate constant $T_{S}^{-1}$ for an exchanging system is given

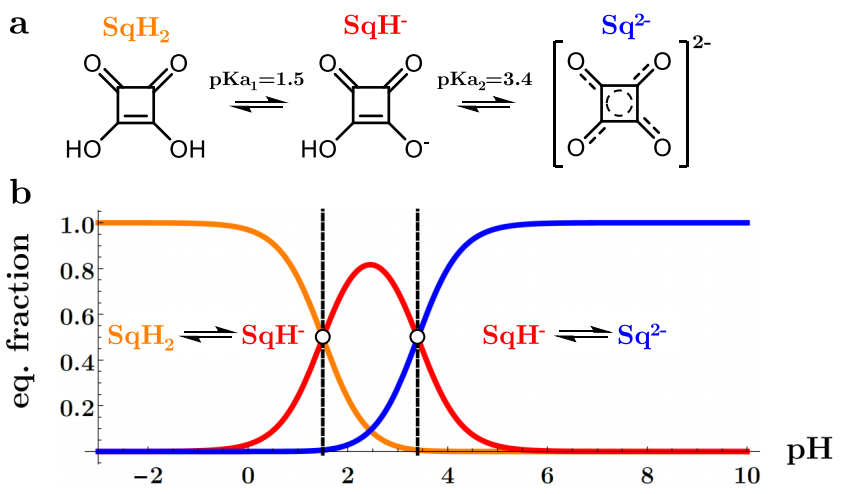

Figure 3. a) The three protonation stages of $1,2-{ }^{13} \mathrm{C}_{2}$-squaric acid. The protonation stages are: squaric acid $\left(\mathrm{SqH}_{2}\right)$, hydrogen squarate $\left(\mathrm{SqH}^{-}\right)$and squarate $\left(\mathrm{Sq}^{2-}\right)$. The dynamical equilibria $\mathrm{SqH}_{2} \rightleftharpoons \mathrm{SqH}^{-}$and $\mathrm{SqH}^{-} \rightleftharpoons \mathrm{Sq}^{2-}$ are characterised by $\mathrm{pKa}$ values of $\mathrm{pKa}_{1}=1.5$ and $\mathrm{pKa}_{2}=3.4$, respectively ${ }^{79}$. b) Equilibrium fractions of the different protonation stages as a function of $\mathrm{pH}$.

by a population-weighted average of the relaxation rate constants for the participating chemical species ${ }^{30}$. This theory is used to show that singlet relaxation may be used to assess the binding of ligands to proteins, with significant biochemical applications ${ }^{30-34}$.

However, the theoretical framework used for ligand binding studies ${ }^{30-34}$ cannot explain the data in figure 2 . If $T_{S}^{-1}$ were given by a population-weighted average of the $T_{S}^{-1}$ values for the participating species, the strongest $\mathrm{pH}$ dependence would be expected in the region of the $\mathrm{pK}_{\mathrm{a}}$ values, i.e. below $\mathrm{pH} \sim 4$, not around $\mathrm{pH} \sim 11$, as is observed.

The data in figure 2 therefore indicate a strong chemical-exchange mechanism for singlet relaxation mechanism that has a much stronger effect than the mere averaging of the rate constant $T_{S}^{-1}$ over the participating species. We propose a mechanism of this kind below. The protonation of a squarate oxygen atom causes a substantial change in the electronic structure which leads in turn to a large isotropic chemical shift difference between the two ${ }^{13} \mathrm{C}$ sites. In high magnetic field the isotropic shift difference of the protonated species, measured in frequency units, greatly exceeds the scalar spin-spin coupling between the two ${ }^{13} \mathrm{C}$ nuclei. Hence, in the protonated form, the singlet state of the ${ }^{13} \mathrm{C}_{2}$ pair is not even an approximate eigenstate of the coherent spin Hamiltonian. This leads to a rapid loss of singlet order upon each protonation event. As discussed below, within a certain kinetic regime, the singlet relaxation rate constant $T_{S}^{-1}$ becomes directly proportional to the rate of protonation of the squarate dianion. The protonation rate is strongly dependent on the hydronium concentration and is therefore $\mathrm{pH}$-dependent.

We provide approximate theoretical expressions for the singlet relaxation rate constant of a spin- $1 / 2$ pair ensemble, using a simplified model of 2-site exchange. Three terms are shown to contribute to the singlet relaxation 
rate constant $T_{S}^{-1}$ in the presence of chemical exchange. The first two terms are the population-weighted averages of the $T_{S}^{-1}$ values for the participating species. These terms correspond closely to the theory developed for ligand binding applications of singlet $\mathrm{NMR}^{30-34}$. Since these terms depend on equilibrium populations, they do not depend on the individual values of the kinetic rate constants, but only on their ratio. The third term is the most important one, in the current context. It depends on the individual kinetic rate constants of the chemical transformations and cannot be described as a populationweighted average of some quantity. We therefore call the third term the kinetic contribution to the singlet relaxation rate constant. Since proton exchange is usually a very fast process, the third term (kinetic term) can easily dominate, even when some of the participating species have negligible equilibrium concentrations. That is the key to understanding the remarkable $\mathrm{pH}$-dependent $T_{S}$ data in figure 2 .

Apart from leading to an understanding of singlet relaxation in systems undergoing chemical exchange, the results described here indicate that nuclear singlet relaxation may be an exquisitely sensitive reporter of rare chemical events. For example, an individual molecule of ${ }^{18} \mathrm{O}$-enriched $1,2-{ }^{13} \mathrm{C}_{2}$ squarate, in a solution with $\mathrm{pH} \sim 11$, only picks up a proton about once a minute on average, and that proton is resident for only a fraction of a millisecond, on average, before returning to the solvent. The ${ }^{13} \mathrm{C}_{2}$ singlet relaxation measurement detects the rare protonation events by means of a significant fall in the $T_{S}$ value. It is hard to find other methods for the reliable characterisation of such infrequent chemical events under equilibrium conditions.

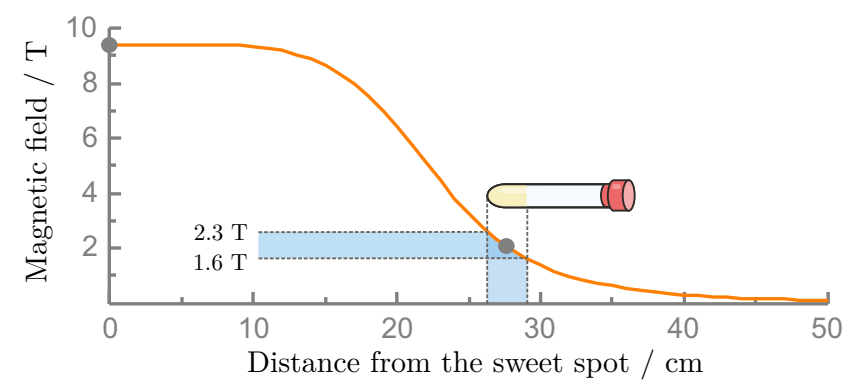

Figure 4. Magnetic fringe field profile of the utilised $400 \mathrm{MHz}$ magnet. Blue area depicts magnetic field distribution experienced by a sample filling $2 \mathrm{~cm}$ of a standard NMR tube. Grey dots indicate the two magnetic fields values selected for the study.

\section{METHODS}

\section{A. Sample preparation}

The synthesis of $1,2-{ }^{13} \mathrm{C}_{2}$ squaric acid (3,4dihydroxycyclobut-3-ene-1,2-dione- $1,2-{ }^{13} \mathrm{C}_{2}$ ) will be described in a following publication. For all experiments $5.8 \mathrm{mg}$ of $1,2-{ }^{13} \mathrm{C}_{2}$ squaric acid $\left[{ }^{16} \mathrm{O}_{4}\right]$ were dissolved in $0.5 \mathrm{~mL}$ of a $1: 1$ mixture of $\mathrm{H}_{2}{ }^{18} \mathrm{O} \mathrm{D}_{2}{ }^{16} \mathrm{O}$ leading to a concentration of $0.1 \mathrm{M}$. The isotopic purity of the $\mathrm{H}_{2}{ }^{18} \mathrm{O}$ was $97.1 \%$. Solutions were incubated at $80{ }^{\circ} \mathrm{C}$ for two hours allowing the ${ }^{16} \mathrm{O} /{ }^{18} \mathrm{O}$ exchange process to reach its dynamical equilibrium. Individual samples were prepared for each $\mathrm{pH}$ value, with the $\mathrm{pH}$ adjusted by step-wise addition of aqueous solutions of $2 \mathrm{M} \mathrm{NaOH}$ (for high $\mathrm{pH}$ ) or $0.2 \mathrm{M} \mathrm{HCl}$ (for low $\mathrm{pH})$. In each instance the total volume added did not exceed $65 \mu \mathrm{L}$ leading to a negligible effect on the sample concentration. The $\mathrm{pH}$ values were estimated by using a $\mathrm{pH}$ meter equipped with a Hamilton SpinTrode probe (www.hamiltoncompany.com), suitable for $\mathrm{pH}$ measurements inside NMR tubes. The calibration of the $\mathrm{pH}$ meter was verified over the full $\mathrm{pH}$ range using standard buffers. The $\mathrm{pH}$ readings of the samples were taken both before and after the NMR measurements. No significant change was observed. For the sake of simplicity, we ignore the potential complications associated with solvent deuteration, such as: (i) the influence of solvent deuteration on the acid-base equilibrium; (ii) the distinction between the concentration of the hydronium ion, indicated by $\mathrm{pH}$, and that of its deuterated analogues; (iii) the modified response of the $\mathrm{pH}$-meter, induced by solvent deuteration. We verified by conventional acid-base titration, using the same solvent composition as for the NMR experiments, that the $\mathrm{pK}_{\mathrm{a}}$ values of squaric acid are 1.3 and 3.4, in good agreement with the literature ${ }^{78}$, indicating that any isotope effects on the acid-base equilibria are minor.

\section{B. Instrumental details}

Experiments were performed on $400 \mathrm{MHz}$ and $700 \mathrm{MHz}$ Bruker Avance Neo systems. For all samples the recycling delays between transients were set to $60 \mathrm{~s}$ corresponding to at least three $T_{1}$ periods. ${ }^{13} \mathrm{C}$ pulse nutation frequencies were given by $\sim 13.9 \mathrm{kHz}$ at $9.4 \mathrm{~T}$, and $\sim 10.7 \mathrm{kHz}$ at 16.5 T. $T_{1}$ measurements used a single transient for each data point. $T_{S}$ measurements (see below) used averaging over 1 to 8 transients in order to achieve a signal-to-noise of $\sim 16$ for the first data point. The NMR signals were sampled with $131 \mathrm{k}$ data points and a spectral width of $146 \mathrm{ppm}$.

Apart from experiments at fixed magnetic fields strengths of $16.5 \mathrm{~T}(700 \mathrm{MHz})$ and $9.4 \mathrm{~T}(400 \mathrm{MHz})$, field cycling experiments were performed inside the fringe field of the $9.4 \mathrm{~T}$ magnet. An overview of the fringe field as a function of the detection coil center to center of the sample tube is given in figure 4. Samples were prepolarised at $9.4 \mathrm{~T}$ before being shuttled to a fringe field region of $1.9 \mathrm{~T}$ as indicated in figure 4 . The sample transport was performed using a stepper motor driven sample carriage operated at a fixed velocity of $1 \mathrm{~m} \mathrm{~s}^{-1}$, as described in reference 80 . Typical shuttle times were on the order of 
$300 \mathrm{~ms}$. Signal detection was performed by shuttling the sample back into the detection region of the high field NMR magnet and applying a $\pi / 2$ pulse.

\section{Pulse sequences}

\section{1. $T_{1}$ measurements}

Longitudinal relaxation time constants at 16.5, 9.4 and 1.9 $\mathrm{T}$ were determined with a standard inversion recovery experiment. For $T_{1}$ measurements at $1.9 \mathrm{~T}$ the sample was first prepolarised at $9.4 \mathrm{~T}$, the magnetisation then inverted and shuttled to $1.9 \mathrm{~T}$ as described above. After a variable recovery period the sample was shuttled back to the detection region of the high field magnet to indirectly observe its recovery to thermal equilibrium at $1.9 \mathrm{~T}$.

\section{2. $T_{S}$ measurements}

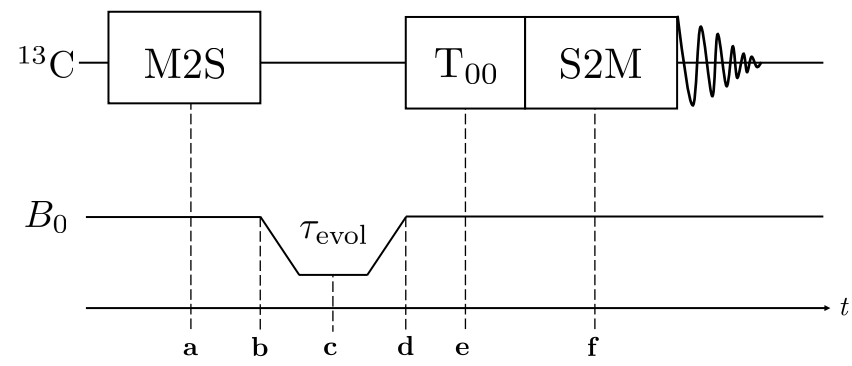

Figure 5. Pulse sequence protocol for $T_{S}$ measurements at variable magnetic strength. Singlet order is excited via an M2S block on the ${ }^{13} \mathrm{C}$ channel (a). After singlet order excitation the sample is shuttled (optional) to a desired magnetic field strength (b) and allowed to evolve for a time period $\tau_{\text {evol }}$ (c). In case of a field cycling experiment the sample is shuttled back into the sweet spot of the detection magnet (d). After the free evolution period unwanted spin operator components are filtered out using a gradient based singlet filtration step (e). Signal detection is initialised by reconverting unobservable singlet order into observable magnetisation via the S2M sequence (f).

An overview of the experimental procedure for $T_{S}$ estimation is given in figure 5 . (a) The initial M2S block (see figure 6a) converts thermally polarised ${ }^{13} \mathrm{C}$ magnetisation into homonuclear ${ }^{13} \mathrm{C}$-singlet order ${ }^{7,8}$. Experimentally optimised M2S parameters for ${ }^{18} \mathrm{O}$-enriched $1,2-{ }^{13} \mathrm{C}_{2}$ squaric acid are given as a function of $\mathrm{pH}$ in table I. (b) After singlet order excitation an optional field cycling step is introduced to transport the sample to a desirable magnetic field strength. (c) Singlet order is allowed to freely evolve for a variable duration $\tau_{\text {evol }}$. (d) For the case of field cycling experiments the sample is shuttled back into the active region of the magnet. (e) Application of a gradient-based singlet order filter (see $\mathbf{a}$

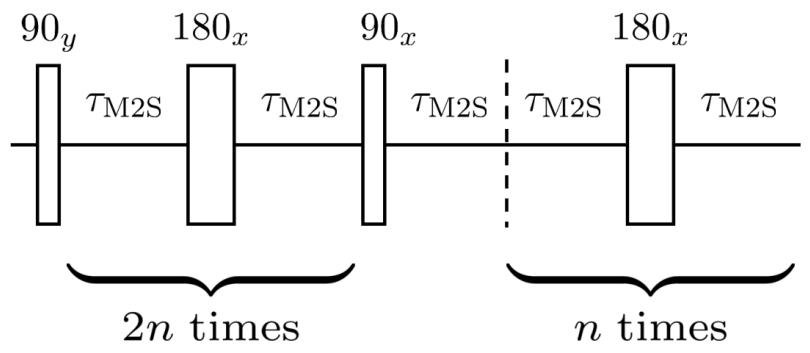

b

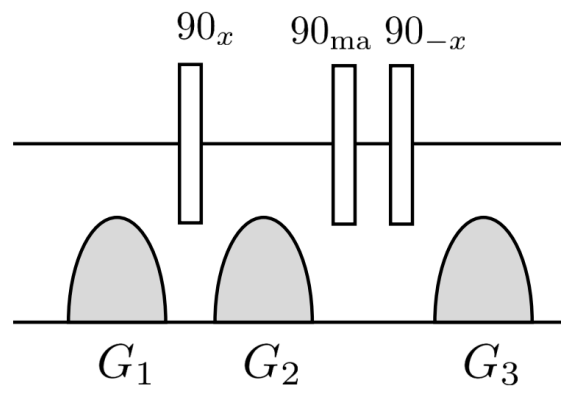

Figure 6. a) Magnetisation-to-Singlet (M2S) pulse sequence. b) Gradient based singlet order filtration scheme ( $\mathrm{T}_{00}$ filter). The abbreviation "ma" refers to the "magic angle" $\simeq 54.7^{\circ}$.

figure $6 \mathrm{~b}$ ) isolates NMR signals passing through singlet order $^{81}$. The field gradient parameters for the singlet filter are summarised in table II. (f) The S2M sequence (the chronological reverse of the M2S sequence) reconverts the singlet order back into observable magnetisation.

In order to compensate for field homogeneity effects, the individual pulses for the M2S and S2M sequence were replaced by composite pulse counterparts. In detail, $180_{\phi}$ pulses were replaced by $90_{90+\phi} 180_{\phi} 90_{90+\phi}$ sequences; $90_{\phi}$ pulses were replaced by $180_{97.2+\phi} 360_{291.5+\phi} 180_{97.2+\phi}$ $90_{\phi}$ sequences ${ }^{82,83}$. The free evolution delays were adjusted to account for the duration of the elongated pulse elements. For example, echo delays were shortened by half the duration of the refocusing element.

\section{RESULTS}

\section{A. ${ }^{13} \mathrm{C}$ spectra}

\section{1. ${ }^{13} \mathrm{C}$ chemical shifts and exchange broadening}

${ }^{13} \mathrm{C}$ NMR spectra of an ${ }^{18} \mathrm{O}$-enriched $1,2-{ }^{13} \mathrm{C}_{2}$ squarate solution for four different $\mathrm{pH}$ values are shown in figure 7 . The ${ }^{13} \mathrm{C}$ chemical shifts show a strong $\mathrm{pH}$-dependence in the regime $\mathrm{pH} \lesssim 7$. The $\mathrm{pH}$-dependence becomes weak above $\mathrm{pH} \sim 7$.

The ${ }^{13} \mathrm{C}$ shifts may be interpreted qualitatively by as- 
Table I. Experimental M2S (S2M) parameters for the central ${ }^{13} \mathrm{C}$ peak of ${ }^{18} \mathrm{O}$-enriched $1,2-{ }^{13} \mathrm{C}_{2}$ squarate dissolved in a $1: 1$ mixture of $\mathrm{H}_{2}{ }^{18} \mathrm{O}: \mathrm{D}_{2}{ }^{16} \mathrm{O}$ as a function of $\mathrm{pH}$.

\begin{tabular}{l|l|l|l|l|l|l|l}
\hline \multicolumn{1}{l}{$400 \mathrm{MHz}$} \\
\hline $\mathrm{pH}$ & 1.3 & 7.2 & 9.9 & 10.9 & 11.9 & 12.7 & 14.0 \\
$n_{\mathrm{M} 2 \mathrm{~S}}$ & 14 & 14 & 14 & 14 & 14 & 14 & 16 \\
$\tau_{\mathrm{M} 2 \mathrm{~S}} / \mathrm{ms}$ & 4.37 & 4.54 & 4.54 & 4.54 & 4.54 & 4.37 & 4.54 \\
\hline $700 \mathrm{MHz}$ \\
\hline $\mathrm{pH}$ & 1.3 & 7.2 & 9.9 & 10.9 & 11.9 & 12.7 & 14.0 \\
$n_{\mathrm{M} 2 \mathrm{~S}}$ & 9 & 9 & 9 & 9 & 9 & 9 & 9 \\
$\tau_{\mathrm{M} 2 \mathrm{~S}} / \mathrm{ms}$ & 4.35 & 4.54 & 4.54 & 4.53 & 4.54 & 4.54 & 4.55
\end{tabular}

Table II. Field gradient specifications for singlet order filtration.

\begin{tabular}{l|l|l|l}
\hline \multicolumn{4}{l}{$400 \mathrm{MHz}$} \\
\hline $\begin{array}{l}\text { gradient strength } \\
\text { duration }\end{array}$ & $-8 \mathrm{G} / \mathrm{cm}-8$ & $-7 \mathrm{G} / \mathrm{cm}$ & $-7.5 \mathrm{G} / \mathrm{cm}$ \\
$2.2 \mathrm{~ms}$ & $1.0 \mathrm{~ms}$ & $4.0 \mathrm{~ms}$ \\
\hline $700 \mathrm{MHz}$ & \multicolumn{4}{|l}{} \\
\hline $\begin{array}{l}\text { gradient strength } \\
\text { duration }\end{array}$ & $9.9 \mathrm{G} / \mathrm{cm}$ & $-4.9 \mathrm{G} / \mathrm{cm}$ & $-4.9 \mathrm{G} / \mathrm{cm}$ \\
& $4.4 \mathrm{~ms}$ & $2.4 \mathrm{~ms}$ & $2.0 \mathrm{~ms}$
\end{tabular}

suming rapid exchange between the protonated and deprotonated forms in figure 3. If the exchange is assumed to be rapid relative to the ${ }^{13} \mathrm{C}$ chemical shift changes, the observed ${ }^{13} \mathrm{C}$ chemical shift corresponds to the mean value for the exchanging species, weighted by their populations. The $\mathrm{pH}$-dependence is weak above $\mathrm{pH} \sim 7$ since the deprotonated $\mathrm{Sq}^{2-}$ species dominates in this regime.

The observed broadening of the ${ }^{13} \mathrm{C}$ spectrum around $\mathrm{pH} \sim 4$ indicates that in this $\mathrm{pH}$ regime, the exchange of

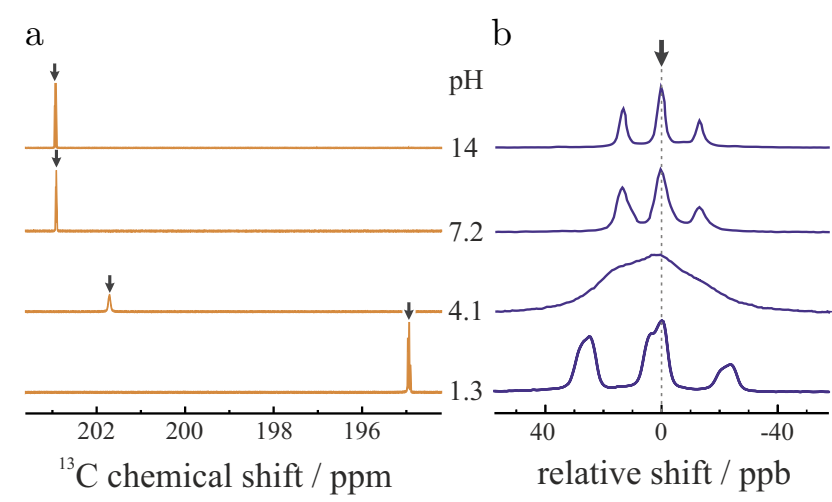

Figure 7. (a) ${ }^{13} \mathrm{C}$ spectra of an ${ }^{18} \mathrm{O}$-enriched $1,2-{ }^{13} \mathrm{C}_{2}$ squarate solution as a function of $\mathrm{pH}$. The spectra were acquired at $16.5 \mathrm{~T}$ after application of a single $\pi / 2$ pulse. (b) Expansions of the relevant spectral regions. the protonated forms occurs on a timescale comparable to the changes in ${ }^{13} \mathrm{C}$ chemical shift frequencies. A quantitative analysis of the $\mathrm{pH}$-dependent ${ }^{13} \mathrm{C}$ chemical shifts and linewidths would require consideration of a complex exchange network with numerous exchanging species and has not been attempted.

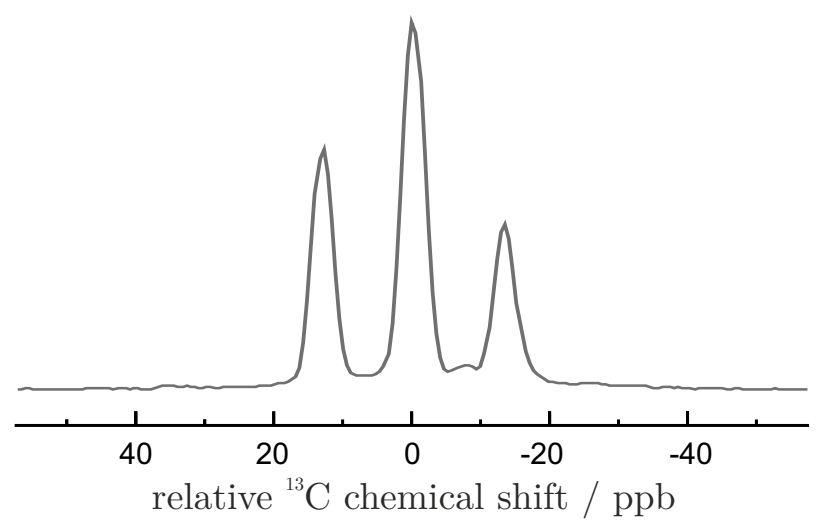

Figure $8 .{ }^{13} \mathrm{C}$ spectrum of $1,2-{ }^{13} \mathrm{C}_{2}$ squarate dissolved in a 1:1 mixture of $\mathrm{H}_{2}{ }^{18} \mathrm{O}: \mathrm{D}_{2}{ }^{16} \mathrm{O}$ at $\mathrm{pH}=14.0$. The spectrum was acquired at $16.5 \mathrm{~T}$ after applying a single $\pi / 2$ pulse. The chemical shift scale is relative to the central peak at 203.05 ppm.

\section{Isotope shifts}

At most $\mathrm{pH}$ values, the ${ }^{13} \mathrm{C}$ spectra display a triplet fine structure due to secondary isotope shifts induced by the randomly distributed ${ }^{18} \mathrm{O}$ nuclei (see figure $7 \mathrm{~b}$ ). A representative ${ }^{13} \mathrm{C}$ equilibrium spectrum of ${ }^{18} \mathrm{O}$-enriched $1,2-{ }^{13} \mathrm{C}_{2}$ squarate at $16.5 \mathrm{~T}$ and $\mathrm{pH}=14.0$ is shown in figure 8 . The spectrum displays three sharp peaks. The two outer peaks are separated by $\simeq-15.0 \mathrm{ppb}$ and $\simeq+15.0 \mathrm{ppb}$ from the central peak. The integrated intensities of the three peaks are in the approximate ratios of $1.2: 2.0: 1.0$.

In this work we follow the convention of an isotope shift being defined as the change in the chemical shift upon substitution of the lighter nucleus $(l)$ by the heavier nucleus $(h)$

$$
{ }^{n} \Delta \mathrm{X}(h)=\delta_{\mathrm{X}}(h)-\delta_{\mathrm{X}}(l),
$$

where $\mathrm{X}$ represents the observed nucleus and $n$ the number of chemical bonds between $\mathrm{X}$ and $h$. This convention was used in a study of ${ }^{18} \mathrm{O}$-enriched oxalate ${ }^{76}$, but differs in sign from the most common convention for secondary isotope shifts (see, for example, Hansen ${ }^{77}$ ).

At high $\mathrm{pH}$, the $\mathrm{Sq}^{2-}$ species dominates. Under these conditions the spectrum may be interpreted in similar fashion to ${ }^{18} \mathrm{O}$-enriched oxalate ${ }^{76}$. The following assumptions are made:

1. Two-bond and three-bond ${ }^{18} \mathrm{O}$-induced isotope shifts are ignored. 
2. The magnitude of one-bond ${ }^{18} \mathrm{O}$-induced isotope shifts is small compared to the $J$-coupling of the ${ }^{13} \mathrm{C}$ pair.

These assumptions allow the ${ }^{13} \mathrm{C}$ spin system of each ${ }^{18} \mathrm{O}$-isotopologues to be classified as either a magnetically equivalent $\mathrm{A}_{2}$ spin system, or a near-equivalent $\mathrm{AB}$ spin system. Each $\mathrm{A}_{2}$ spin systems gives rise to a single ${ }^{13} \mathrm{C}$ peak, while in general, each near-equivalent $\mathrm{AB}$ system gives rise to a four-peak subspectrum with two strong central peaks and two weak outer peaks. However, under the conditions that apply here, the outer peaks have small intensity, and the two inner peaks are unresolved and coalesce to a single peak at the mean of the two ${ }^{13} \mathrm{C}$ chemical shifts. The experimentally observed spectral pattern may therefore be understood as a superposition of these single ${ }^{13} \mathrm{C}$ resonances (see figure 9 ).

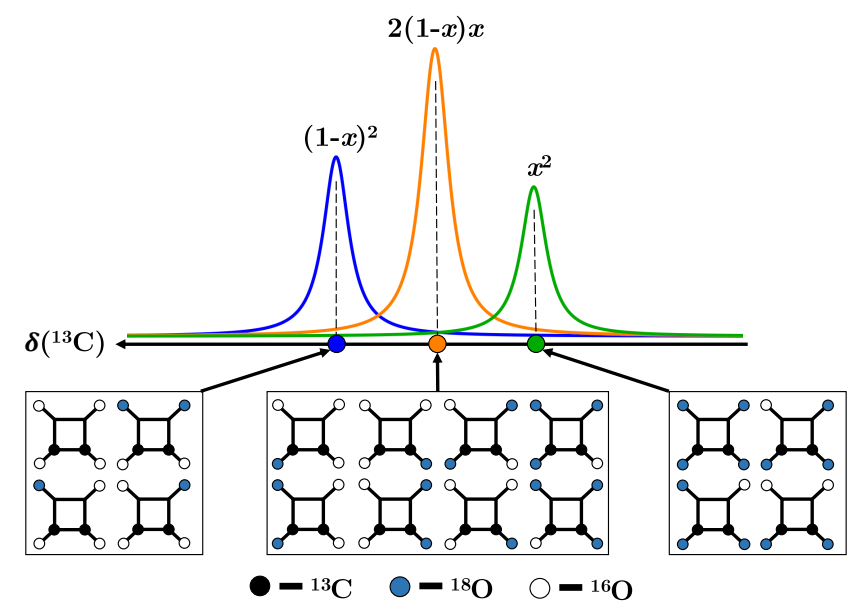

Figure 9. Qualitative interpretation of the experimentally observed spectral pattern in figure 8 . The three resonances may be attributed to isotopologues having zero, one or two ${ }^{18} \mathrm{O}$ atoms directly bound to the ${ }^{13} \mathrm{C}$ spin pair. The grouping of all possible ${ }^{18} \mathrm{O} 1,2-{ }^{13} \mathrm{C}_{2}$ squarate isotopologues is shown below. The relative intensities of the peaks are proportional to the relative abundance of a given group.

The observed mean chemical shifts of the different peaks are thus given by

$$
\begin{aligned}
& \left\langle\delta_{\text {low }}\right\rangle=\delta_{0}, \\
& \left\langle\delta_{\text {central }}\right\rangle=\delta_{0}+{ }^{1} \Delta^{13} \mathrm{C}\left({ }^{18} \mathrm{O}\right) / 2, \\
& \left\langle\delta_{\text {high }}\right\rangle=\delta_{0}+{ }^{1} \Delta^{13} \mathrm{C}\left({ }^{18} \mathrm{O}\right),
\end{aligned}
$$

where ${ }^{1} \Delta^{13} \mathrm{C}\left({ }^{18} \mathrm{O}\right)$ represents the ${ }^{18} \mathrm{O}$-induced singlebond isotope shift. The spectral separation between the high- $\delta$ and low- $\delta$ peak is therefore equal to the one-bond ${ }^{18} \mathrm{O}$-induced isotope shift experienced by a ${ }^{13} \mathrm{C}$ nucleus. At high $\mathrm{pH}$ values the experimental data (figure 8) give the following estimate of the one-bond ${ }^{18} \mathrm{O}$-induced isotope shift: ${ }^{1} \Delta^{13} \mathrm{C}\left({ }^{18} \mathrm{O}\right) \simeq-15 \mathrm{ppb}$.

The intensities of the different peaks are proportional to the abundances of the different isotopologues. From simple statistical arguments, assuming a random distribution of ${ }^{18} \mathrm{O}$ nuclei amongst the oxygen atoms, the abundances of the species giving rise to the three peaks are proportional to $\left\{(1-x)^{2}, 2 x(1-x), x^{2}\right\}$, where $x$ is the abundance of ${ }^{18} \mathrm{O}$ nuclei, and $1-x$ is the abundance of ${ }^{16} \mathrm{O}$ nuclei. The observed spectral amplitudes are consistent with an ${ }^{18} \mathrm{O}$ enrichment level of $x \simeq 46 \%$. This corresponds well to the known composition of the solvent (see section II A).

The ${ }^{18} \mathrm{O}$ isotope shifts become significantly larger at low $\mathrm{pH}$, as shown in figure $7(\mathrm{~b})$. This effect has not been analyzed in detail, but is provisionally attributed to small changes of the equilibrium fractions of the different protonated species upon ${ }^{18} \mathrm{O}$ substitution. ${ }^{18} \mathrm{O}$ substitution changes the zero-point vibrational energy of - $\mathrm{OH}$ bonds and therefore influences the equilibrium fractions of the various protonated species, which leads in turn to a perturbation of the ${ }^{13} \mathrm{C}$ chemical shifts when averaged over all exchanging species. This mechanism has been identified as the source of relatively large and remote ${ }^{18} \mathrm{O}$-induced isotope shifts in the ${ }^{13} \mathrm{C}$ spectra of some carboxylic acids ${ }^{84,85}$.

\section{B. Spin-lattice relaxation}

The ${ }^{13} \mathrm{C} T_{1}$ values of ${ }^{18} \mathrm{O}$-enriched $1,2-{ }^{13} \mathrm{C}_{2}$ squaric acid at 2, 9.4 and $16.5 \mathrm{~T}$ as a function of $\mathrm{pH}$ are shown in figure 10 .

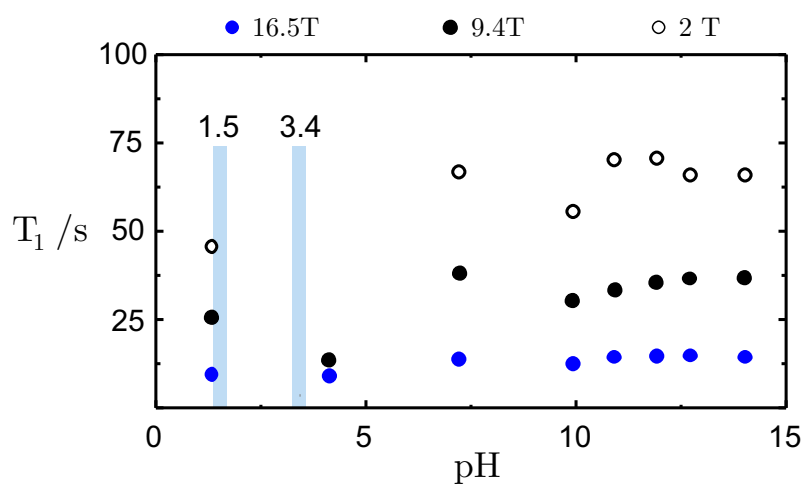

Figure 10. Longitudinal decay time constants for ${ }^{18} \mathrm{O}$ enriched $1,2-{ }^{13} \mathrm{C}_{2}$ squaric acid in $\mathrm{H}_{2}{ }^{18} \mathrm{O}: \mathrm{D}_{2}{ }^{16} \mathrm{O}$ solution as a function of $\mathrm{pH}$ at 2, 9.4 and $16.5 \mathrm{~T}$. The blue bars indicate the $\mathrm{pK}_{\mathrm{a}}$ values for the chemical equilibria between squaric acid $\rightleftharpoons$ hydrogen-squarate and hydrogen-squarate $\rightleftharpoons$ sqarate.

In contrast to the observed $T_{S}$ values, only a weak $\mathrm{pH}$ dependence of $T_{1}$ is observed. For example, at $9.4 \mathrm{~T}$ we observe $T_{1}$ values of approximately $30 \mathrm{~s}$ for all $\mathrm{pH}$ values apart from $\mathrm{pH}=4.1$, where $T_{1}$ decreases to $\approx 15 \mathrm{~s}$. In particular, $T_{1}$ does not display any notable features either at the $\mathrm{pK}_{\mathrm{a}}$ values of squaric acid, nor at the inflection point $\mathrm{pH} \simeq 10$ at which the observed $T_{S}$ values start to drastically increase. 
Similar observations are made for the $\mathrm{pH}$-dependent $T_{1}$ values at $16.5 \mathrm{~T}$ and $2 \mathrm{~T}$ with an overall decrease in $T_{1}$ as the magnetic field strength increases. This is consistent with a dominant CSA relaxation mechanism, proportional to the square of the magnetic field strength.

\section{Singlet NMR}

\section{Singlet order excitation}

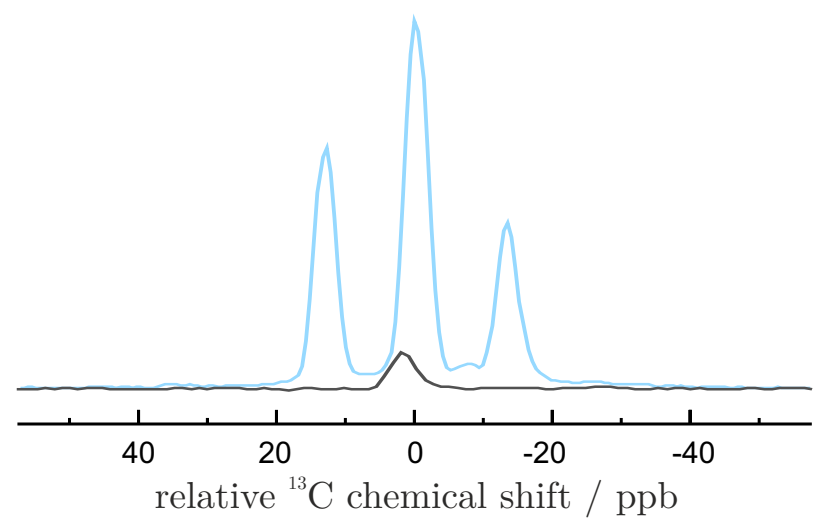

Figure 11. Singlet filtered ${ }^{13} \mathrm{C}$ spectrum of ${ }^{18} \mathrm{O}$-enriched $1,2-$ ${ }^{13} \mathrm{C}_{2}$ squarate dissolved in a $1: 1$ mixture of $\mathrm{H}_{2}{ }^{18} \mathrm{O}: \mathrm{D}_{2}{ }^{16} \mathrm{O}$ squarate at $\mathrm{pH}=14.0$ (black line). The spectrum was acquired at $16.5 \mathrm{~T}$ using the pulse sequence protocol shown in figure 5 with no evolution delay. The blue line shows the spectrum after application of a single $\pi / 2$ pulse. Both spectra are referenced to a carrier frequency of $203.05 \mathrm{ppm}$.

As shown in figure 9, the central peak of the ${ }^{13} \mathrm{C}$ triplet is associated with $1,2-{ }^{13} \mathrm{C}_{2}$-squarate species with an ${ }^{18} \mathrm{O}$ atom attached to one ${ }^{13} \mathrm{C}$ atom and a ${ }^{16} \mathrm{O}$ atom attached to the other. These species contain strongly coupled ${ }^{13} \mathrm{C}$ pairs with a small chemical shift difference between the two ${ }^{13} \mathrm{C}$ nuclei, and are suitable candidates for singlet order excitation techniques. Figure 11 shows a singlet-filtered ${ }^{18} \mathrm{O}$-enriched $1,2-{ }^{13} \mathrm{C}_{2}$ squarate spectrum at $\mathrm{pH}=14.0$. The spectrum was acquired at $16.5 \mathrm{~T}$ using the pulse sequence protocol in figure 5 with the M2S parameters given in table I. The signals from the outer peaks of the triplet are suppressed in the singlet-filtered ${ }^{13} \mathrm{C}$ spectrum since these signals are generated by isotopologues with no ${ }^{18} \mathrm{O}$ atoms directly bonded to both ${ }^{13} \mathrm{C}$ atoms, or with no ${ }^{16} \mathrm{O}$ atoms directly bonded to both ${ }^{13} \mathrm{C}$ atoms. Although these species include isotopologues with asymmetric distributions of ${ }^{18} \mathrm{O}$ atoms on the other side of the molecule to the ${ }^{13} \mathrm{C}_{2}$ pair, these remote heavy atom substitutions are too far away to allow appreciable excitation of ${ }^{13} \mathrm{C}$ singlet order, for the chosen pulse sequence parameters.

Depending on the $\mathrm{pH}$ of the solution the intensity of the singlet filtered peak displays approximately $10 \%$ to $30 \%$ of the intensity of the unfiltered spectrum. The small frequency shift of the singlet-filtered spectrum with respect to the pulse-acquire spectrum is attributed to a slight change in sample temperature between experiments.

The experimentally optimised M2S pulse sequence parameters (see table I) serve as an independent estimator for the ${ }^{1} \Delta^{13} \mathrm{C}\left({ }^{18} \mathrm{O}\right)$ isotope shift and the scalar coupling constant $J_{12}$. In the absence of relaxation losses the relationship between the optimal M2S parameters and the spin interaction parameters are given by ${ }^{8,86}$ :

$$
\begin{aligned}
& n_{\mathrm{M} 2 \mathrm{~S}}=\operatorname{round}\left(\pi J_{12} / 4(\Delta \Omega / 2 \pi)\right), \\
& \tau_{\mathrm{M} 2 \mathrm{~S}}=1 /\left(4 \sqrt{J_{12}^{2}+(\Delta \Omega / 2 \pi)^{2}}\right),
\end{aligned}
$$

where $\Delta \Omega$ is the resonance frequency difference between the two spins in $\mathrm{rad} \mathrm{s}^{-1}$. Inversion of these relations leads to an estimate of $J_{12} \simeq 54.86 \pm 0.5 \mathrm{~Hz}$ for the scalar coupling constant. The resonance frequency difference $\Delta \Omega$ is consistent with the ${ }^{18} \mathrm{O}$-induced isotope shift estimated from the spectral splittings (see section III A).

The experimentally optimised M2S parameters summarised in table I indicate that similar estimates are obtained for the majority of the other $\mathrm{pH}$ values. The only exception is the $\mathrm{pH}$ region $2<\mathrm{pH}<7$, where we were unable to observe any singlet-filtered signal components, presumably due to short $T_{2}$ values associated with the exchange broadening at intermediate $\mathrm{pH}$ which is readily visible in figure 7 . The short $T_{2}$ values cause strong signal losses during the M2S/S2M pulse schemes.

\section{Singlet decay time constants}

The pulse sequence protocol shown in figure 5 was used to study nuclear singlet decay time constants of ${ }^{18} \mathrm{O}$ enriched $1,2-{ }^{13} \mathrm{C}_{2}$ squaric acid at a set of $\mathrm{pH}$ values and magnetic fields. Figure 2 summarises the nuclear singlet decay time constants as a function of $\mathrm{pH}$ at 16.5, 9.4 and $2 \mathrm{~T}$.

At all magnetic fields, the $T_{S}$ values of ${ }^{18} \mathrm{O}$-enriched 1,2- ${ }^{13} \mathrm{C}_{2}$ squaric acid vary strongly with the $\mathrm{pH}$ value of the solution. The overall $\mathrm{pH}$ dependence resembles a sigmoidal curve. For example, at $9.4 \mathrm{~T}$ the nuclear singlet decay time constant of ${ }^{18} \mathrm{O}$-enriched $1,2-{ }^{13} \mathrm{C}_{2}$ squaric acid at $\mathrm{pH}=1.3$ is given by $T_{S}=18.3 \mathrm{~s}$. Initially, increasing the $\mathrm{pH}$ does not lead to any significant changes in $T_{S}$. However at a $\mathrm{pH}$ value of $\sim 11$ a sharp increase in $T_{S}$ is observed reaching values up to $T_{S}=53 \mathrm{~s}$. The maximum $T_{S}$ value of $275 \mathrm{~s}$ is reached for $\mathrm{pH}=14.0$.

Similar behaviour is observed at $16.5 \mathrm{~T}$ and $2 \mathrm{~T}$ with an overall increase in relaxation times as the magnetic field strength decreases. For example, at $16.5 \mathrm{~T}, T_{S}$ reaches a maximum of $T_{S}=121 \mathrm{~s}$, whereas at $2 \mathrm{~T}$ the observer singlet decay time constant reaches a maximum of $T_{S}=380 \mathrm{~s}$. The increase in $T_{S}$ with a decrease in the magnetic field strength is consistent with a dominant chemical shift anisotropy relaxation mechanism in this $\mathrm{pH}$ range. 


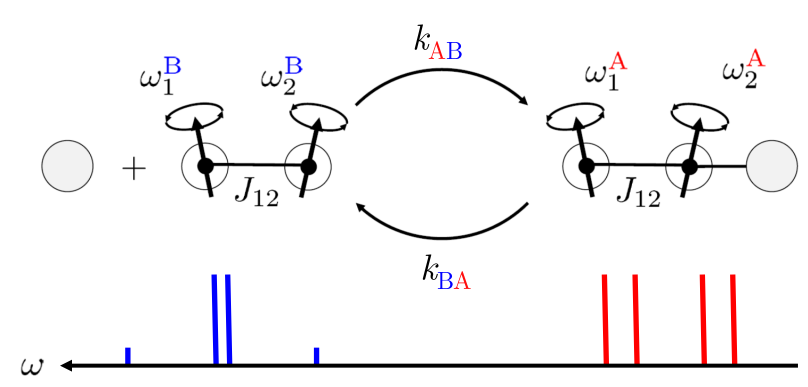

Figure 12. Toy model for nuclear singlet relaxation via chemical exchange events. The model consists of two chemically exchanging configurations A (red) and B (blue) connected via the exchange rate constants $k_{\mathrm{BA}}$ (for the conversion of $\mathrm{A}$ to $\mathrm{B}$ ) and $k_{\mathrm{AB}}$ (for the conversion of $\mathrm{B}$ to A). Each configuration is characterised by its own Hamiltonian. For simplicity the toy model assumes the scalar coupling $J_{12}$ to be identical for both configurations. The resonance frequencies $\omega_{j}^{\mathrm{A}}$ and $\omega_{j}^{\mathrm{B}}$ for spins $j \in\{1,2\}$ are in general different for configurations A and B. The spectral patterns for species A and B are indicated in the absence of chemical exchange.

As pointed out above, one of the most surprising features of the $\mathrm{pH}$-dependence of $T_{S}$ is that the transition from fast to slow relaxation occurs around the $\mathrm{pH}$-value of 11 , which is far above the $\mathrm{pK}_{\mathrm{a}}$ values of the acid-base equilibria for this system.

\section{THEORY}

\section{A. Two-site toy model}

A complete description of proton-exchange induced nuclear singlet relaxation of ${ }^{18} \mathrm{O}$-enriched $1,2-{ }^{13} \mathrm{C}_{2}$ squaric acid is made complicated by the large number of exchanging species. Fortunately the basic principles may be illustrated by considering the two-site exchange toy model shown in figure 12 .

The two spins supporting the nuclear singlet state may find themselves in two different molecular environments A (red) and B (blue). This change may be induced upon binding of an otherwise NMR inactive partner (grey ball). The exchange dynamics are described by an exchange matrix $K$

$$
K=\left[\begin{array}{rr}
-k_{\mathrm{BA}} & k_{\mathrm{AB}} \\
k_{\mathrm{BA}} & -k_{\mathrm{AB}}
\end{array}\right] .
$$

The spin Hamiltonians are in general different for configuration $\mathrm{A}$ and $\mathrm{B}$. For simplicity we assume that the scalar coupling constant $J_{12}=\omega_{J} /(2 \pi)$ is identical for both configurations.

Note that the subscripts in symbols such as $k_{\mathrm{BA}}$ should be read from right to left, i.e. $k_{\mathrm{BA}}$ denotes the rate constant for the chemical transformation of species A into $\mathrm{B}$, and vice versa for $k_{\mathrm{AB}}$.
In the following discussion, we use the symbol $\mathbb{X}$ to denote a general chemical species, where $\mathbb{X} \in\{\mathrm{A}, \mathrm{B}\}$ for 2 -site exchange.

The coherent dynamics of a spin system in chemical species $\mathbb{X}$ are governed by the following Hamiltonian:

$$
\begin{aligned}
H_{\mathrm{coh}}^{\mathbb{X}} & =\Omega_{1}^{\mathbb{X}} I_{1 z}+\Omega_{2}^{\mathbb{X}} I_{2 z}+\omega_{J} \mathbf{I}_{1} \cdot \mathbf{I}_{2} \\
& =\bar{\Omega}^{\mathbb{X}} I_{z}+\frac{1}{2} \Delta \Omega^{\mathbb{X}}\left(I_{1 z}-I_{2 z}\right)+\omega_{J} \mathbf{I}_{1} \cdot \mathbf{I}_{2} .
\end{aligned}
$$

The mean (rotating frame) resonance frequency of the spin pair is denoted $\bar{\Omega}^{\mathbb{X}}=\frac{1}{2}\left(\Omega_{1}^{\mathbb{X}}+\Omega_{2}^{\mathbb{X}}\right)$. The resonance frequency difference between the two spins in configuration $\mathbb{X}$ is given by $\Delta \Omega^{\mathbb{X}}=\Omega_{1}^{\mathbb{X}}-\Omega_{2}^{\mathbb{X}}$. At a magnetic field $B$, this is given by

$$
\Delta \Omega^{\mathbb{X}}=-\gamma B \Delta \delta^{\mathbb{X}}
$$

where $\Delta \delta^{\mathbb{X}}$ is the difference in chemical shifts of the two ${ }^{13} \mathrm{C}$ sites in configuration $\mathbb{X}$.

The Hamiltonians $H_{\mathrm{coh}}^{\mathrm{A}}$ and $H_{\mathrm{coh}}^{\mathrm{B}}$ may be used to define the site-specific parts of the coherent Liouvillian

$$
\hat{L}_{\mathrm{coh}}^{\mathbb{X}}=-i \hat{H}_{\mathrm{coh}}^{\mathbb{X}}
$$

where $\hat{H}^{\mathbb{X}}$ is the commutation superoperator for Hamiltonian $H^{\mathbb{X}}$. In general, between exchange events the subensembles A and B evolve under a site-specific Liouvillian $\hat{L}^{\mathbb{X}}$ including coherent and incoherent parts

$$
\hat{L}^{\mathbb{X}}=\hat{L}_{\mathrm{coh}}^{\mathbb{X}}+\hat{\Gamma}^{\mathbb{X}}
$$

where $\hat{\Gamma}^{\mathbb{X}}$ represents a site-specific relaxation superoperator, discussed below.

The evolution of the sub-ensembles A and B including exchange events may be described by coupling their site-specific evolution via the forward and backward rates $k_{\mathrm{BA}}$ and $k_{\mathrm{AB}}{ }^{87-89}$. The resulting equations of motion are given by

$$
\begin{aligned}
& \frac{d}{d t} \rho^{\mathrm{A}}(t)=\left(\hat{L}^{\mathrm{A}}-k_{\mathrm{BA}}\right) \rho^{\mathrm{A}}(t)+k_{\mathrm{AB}} \rho^{\mathrm{B}}(t), \\
& \frac{d}{d t} \rho^{\mathrm{B}}(t)=\left(\hat{L}^{\mathrm{B}}-k_{\mathrm{AB}}\right) \rho^{\mathrm{B}}(t)+k_{\mathrm{BA}} \rho^{\mathrm{A}}(t) .
\end{aligned}
$$

The evolution of $\rho^{\mathrm{A}}$ and $\rho^{\mathrm{B}}$ are conveniently combined by introducing a joint density operator

$$
\sigma(t)=\left[\begin{array}{l}
\rho^{\mathrm{A}}(t) \\
\rho^{\mathrm{B}}(t)
\end{array}\right]
$$

and a joint generator of motion $\hat{G}$

$$
\hat{G}=\hat{L}+\hat{K}=\left[\begin{array}{cc}
\hat{L}^{\mathrm{A}} & 0 \\
0 & \hat{L}^{\mathrm{B}}
\end{array}\right]+\left[\begin{array}{rr}
-k_{\mathrm{BA}} & k_{\mathrm{AB}} \\
k_{\mathrm{BA}} & -k_{\mathrm{AB}}
\end{array}\right] .
$$

Equation 9 then reduces to a single system

$$
\frac{d}{d t} \sigma(t)=\hat{G} \sigma(t),
$$

and the evolution of the combined ensemble is completely characterised by $\hat{G}$. 


\section{B. Site-specific Relaxation Superoperator}

The site-specific relaxation superoperator $\hat{\Gamma}^{\mathbb{X}}$ is typically derived from a microscopic model and captures details about the fluctuating part of the spin Hamilto$\operatorname{nian}^{64}$. For isolated spin-1/2 pairs stochastic modulations of the dipolar and chemical shift interactions are the dominant contributions to the fluctuating Hamiltonian. Any additional relaxation features are approximated by coupling the nuclear spins to an external random field 90-92

$$
\hat{\Gamma}^{\mathbb{X}}=\hat{\Gamma}_{\mathrm{dd}}^{\mathbb{X}}+\hat{\Gamma}_{\mathrm{csa}}^{\mathbb{X}}+\hat{\Gamma}_{\mathrm{erf}}^{\mathbb{X}}
$$

Within the fast motion and isotropic diffusion limit the site-specific relaxation superoperator for dipolar driven spin relaxation takes the form

$$
\begin{aligned}
& \hat{\Gamma}_{\mathrm{dd}}^{\mathbb{X}}=-\xi_{\mathrm{dd}}(\mathbb{X}) \sum_{m=-2}^{+2}(-1)^{m} \hat{T}_{2 m}^{12}(\mathbb{X}) \hat{T}_{2-m}^{12}(\mathbb{X}) \\
& \xi_{\mathrm{dd}}(\mathbb{X})=\frac{6}{5}\left(b_{12}^{\mathbb{X}}\right)^{2} \tau_{\mathrm{c}}^{\mathbb{X}}
\end{aligned}
$$

The rotational correlation time and dipole-dipole spinspin coupling constant (in $\mathrm{rads}^{-1}$ ) for a molecule in configuration $\mathbb{X}$ are denoted by $\tau_{\mathrm{c}}^{\mathbb{X}}$ and $b_{12}^{\mathbb{X}}$ respectively. The operators $T_{2 m}^{12}(\mathbb{X})$ are site-specific second-rank spherical tensor operators resulting from the coupling of the two spin angular momentum operators ${ }^{92}$.

In general, the equilibration of the spin system with a finite-temperature environment should be taken into account by replacing the double commutators in equation 14, and similar equations, by thermally weighted Lindbladian dissipators ${ }^{93}$. This complication is not relevant to the current discussion and has been avoided.

The chemical shift anisotropy tensors are in general different for spin systems in configurations A and B since the electronic environment of the spins is altered upon a binding event. To simplify the discussion we assume axially symmetric chemical shift tensors for both sites, $\eta^{i}(\mathbb{X})=0 . \mathbb{X} \in\{\mathrm{A}, \mathrm{B}\}$. The corresponding relaxation superoperator may be expressed as follows

$$
\begin{aligned}
& \hat{\Gamma}_{\mathrm{csa}}^{\mathbb{X}}=-\sum_{m=-2}^{+2}\left\{\sum_{i=1}^{2}(-1)^{m} \xi_{\mathrm{csa}}^{i}(\mathbb{X}) \hat{X}_{2 m}^{i}(\mathbb{X}) \hat{X}_{2-m}^{i}(\mathbb{X})\right. \\
& \left.+\xi_{\mathrm{csa}}^{i j}(\mathbb{X}) d_{00}^{2}\left(\beta_{P P}^{\mathbb{X}}\right) \sum_{i \neq j}^{2}(-1)^{m} \hat{X}_{2 m}^{i}(\mathbb{X}) \hat{X}_{2-m}^{j}(\mathbb{X})\right\}, \\
& \xi_{\mathrm{csa}}^{i}(\mathbb{X})=\frac{3}{10}\left(\omega_{\mathrm{csa}}^{i}(\mathbb{X})\right)^{2} \tau_{\mathrm{c}}^{\mathbb{X}}, \\
& \xi_{\mathrm{csa}}^{i j}(\mathbb{X})=\sqrt{\xi_{\mathrm{csa}}^{i}(\mathbb{X}) \xi_{\mathrm{csa}}^{j}(\mathbb{X})} \text {. }
\end{aligned}
$$

The frequency $\omega_{\mathrm{csa}}^{i}(\mathbb{X})=-\gamma B_{0} \delta_{\mathrm{csa}}^{i}(\mathbb{X})$ represents the chemical shift anisotropy parameter in $\mathrm{rad} \mathrm{s}^{-1}$. The cross terms are weighted by the second rank reduced Wigner matrix element

$$
d_{00}^{2}(\beta)=\frac{1}{2}\left(3 \cos ^{2} \beta-1\right)
$$

which in turn depends on the angle between the unique principal axes of the two CSA tensors in configuration $\mathbb{X}$, denoted $\beta_{P P}^{\mathbb{X}}$, and the geometric mean of the anisotropy parameters $\xi_{\text {csa }}^{i}(\mathbb{X})$. The operators $X_{2 m}^{i}(\mathbb{X})$ are defined by

$$
X_{2 m}^{i}(\mathbb{X})=\sum_{n=-1}^{+1} C_{101 n}^{2 m} T_{1 n}^{i}(\mathbb{X})
$$

These operators are components of the irreducible spherical tensor resulting from coupling the spherical tensor spin operators with the spherical components of the static magnetic field ${ }^{92}$. The symbols $C_{j m j^{\prime} m^{\prime}}^{J M}$ denote ClebschGordan coefficients.

An external random-field contribution to the relaxation superoperator is often included to take into account unspecified mechanisms, such as interactions with paramagnetic species in solution, or internuclear dipole-dipole couplings with other magnetic nuclei within the same molecule or in different molecules. The corresponding site-specific relaxation superoperator is given by ${ }^{90-92}$

$$
\begin{aligned}
& \hat{\Gamma}_{\mathrm{erf}}^{\mathbb{X}}=-\xi_{\mathrm{erf}}(\mathbb{X})\left\{\sum_{i=1}^{2} \sum_{m=-2}^{+2}(-1)^{m} \hat{T}_{1 m}^{i}(\mathbb{X}) \hat{T}_{1-m}^{i}(\mathbb{X})\right. \\
&\left.+\kappa^{\mathbb{X}} \sum_{i \neq j}^{2} \sum_{m=-2}^{+2}(-1)^{m} \hat{T}_{1 m}^{i}(\mathbb{X}) \hat{T}_{1-m}^{\mathbb{X}}(\mathbb{X})\right\} \\
& \xi_{\mathrm{erf}}(\mathbb{X})=\left(\omega_{\mathrm{erf}}^{\mathbb{X}}\right)^{2} \tau_{\mathrm{erf}}^{\mathbb{X}},
\end{aligned}
$$

where $\tau_{\mathrm{erf}}^{\mathbb{X}}$ describes the correlation time of the random field fluctuations, $\omega_{\text {erf }}^{\mathbb{X}}$ denotes the root-mean-square amplitude in $\operatorname{rad~s}^{-1}$, and $-1 \leq \kappa^{\mathbb{X}} \leq 1$ the correlation coefficient of the fluctuations experienced by spins 1 and 2 , in configuration $\mathbb{X}$. 


\section{Analytical relaxation rate constants}

An analytic approximation to the singlet order decay rate constant under chemical exchange may be constructed by considering the eigenvalues of the join generator of motion $\hat{G}$. For a two-site exchange model the matrix representation of $\hat{G}$ is of dimension $\operatorname{dim}(\hat{G})=2 \times N_{\mathrm{H}}^{2}$, where $N_{\mathrm{H}}$ is the dimension of the underlying Hilbert space. But as discussed in appendix $\mathrm{B}$ the singlet order dynamics for the combined ensemble $(\mathrm{A}+\mathrm{B})$ are well approximated by the following $4 \times 4$ matrix sub-block

$$
\mathbf{g}=\left[\begin{array}{cccc}
\mathrm{SO}^{\mathrm{A}} & O_{\Delta}^{\mathrm{A}} & \mathrm{SO}^{\mathrm{B}} & O_{\Delta}^{\mathrm{B}} \\
\hline-k_{\mathrm{AB}}-R_{S}^{0}(\mathrm{~A}) & i 3^{-1 / 2} \Delta \Omega^{\mathrm{A}} & \sqrt{k_{\mathrm{AB}} k_{\mathrm{BA}}} & 0 \\
i 3^{-1 / 2} \Delta \Omega^{\mathrm{A}} & -k_{\mathrm{AB}}-R_{\Delta}^{0}(\mathrm{~A})+i \omega_{J} & 0 & \sqrt{k_{\mathrm{AB}} k_{\mathrm{BA}}} \\
\sqrt{k_{\mathrm{AB}} k_{\mathrm{BA}}} & 0 & -k_{\mathrm{BA}}-R_{S}^{0}(\mathrm{~B}) & i 3^{-1 / 2} \Delta \Omega^{\mathrm{B}} \\
0 & \sqrt{k_{\mathrm{AB}} k_{\mathrm{BA}}} & i 3^{-1 / 2} \Delta \Omega^{\mathrm{B}} & -k_{\mathrm{BA}}-R_{\Delta}^{0}(\mathrm{~B})+i \omega_{J}
\end{array}\right] .
$$

The sub-block $\mathbf{g}$ is spanned by the operators

$$
\begin{aligned}
& \mathrm{SO}^{\mathbb{X}}=3^{-1 / 2}\left(2 I_{1 z}^{\mathbb{X}} I_{2 z}^{\mathbb{X}}+I_{1-}^{\mathbb{X}} I_{2+}^{\mathbb{X}}+I_{1+}^{\mathbb{X}} I_{2-}^{\mathbb{X}}\right), \\
& O_{\Delta}^{\mathbb{X}}=\frac{1}{2}\left(I_{2 z}^{\mathbb{X}}-I_{1 z}^{\mathbb{X}}+I_{1-}^{\mathbb{X}} I_{2+}^{\mathbb{X}}-I_{1+}^{\mathbb{X}} I_{2-}^{\mathbb{X}}\right) .
\end{aligned}
$$

The operators $\mathrm{SO}^{\mathbb{X}}$ represent singlet order for spin systems in configuration A or B, whereas the operators $O_{\Delta}^{\mathbb{X}}$ represent zero-quantum operators that are anti-symmetric under spin permutation.

The terms $R_{S}^{0}(\mathbb{X})$ and $R_{\Delta}^{0}(\mathbb{X})$ in equation 19 are given by

$$
\begin{aligned}
& R_{S}^{0}(\mathbb{X})=4\left(1-\kappa^{\mathbb{X}}\right) \xi_{\text {erf }}(\mathbb{X})+\frac{10}{9}\left(\xi_{\text {csa }}^{1}(\mathbb{X})+\xi_{\text {csa }}^{2}(\mathbb{X})-2 \xi_{\text {csa }}^{12}(\mathbb{X}) d_{00}^{2}\left(\beta_{P P}^{\mathbb{X}}\right),\right. \\
& R_{\Delta}^{0}(\mathbb{X})=\left(3-\kappa^{\mathbb{X}}\right) \xi_{\text {erf }}(\mathbb{X})+\frac{5}{12} \xi_{\text {dd }}(\mathbb{X})+\frac{5}{6}\left(\xi_{\text {csa }}^{1}(\mathbb{X})+\xi_{\text {csa }}^{2}(\mathbb{X})\right)-\frac{2}{3} \xi_{\text {csa }}^{12}(\mathbb{X}) d_{00}^{2}\left(\beta_{P P}^{\mathbb{X}}\right)
\end{aligned}
$$

We introduce the following symbol for a weighted average over the equilibrium populations of the exchange process:

$$
\begin{aligned}
\langle\langle a, b\rangle\rangle & =p_{\mathrm{eq}}^{\mathrm{A}} a+p_{\mathrm{eq}}^{\mathrm{B}} b, \\
& =\left(k_{\mathrm{AB}} a+k_{\mathrm{BA}} b\right) /\left(k_{\mathrm{BA}}+k_{\mathrm{AB}}\right) .
\end{aligned}
$$

Appendix B gives a perturbative treatment of two-site exchange under the assumptions $k_{\mathrm{BA}} \gg \omega_{J}$ and $\mathrm{pH}>$ $\mathrm{pK}_{a}$, and shows that the decay constant $R_{S}$ for singlet order may be approximated by

$$
R_{S} \simeq R_{S}^{0}+R_{S}^{\mathrm{leak}}+R_{S}^{\mathrm{kin}}
$$

The first term is given by the population-weighted average of the $R_{S}^{0}$ terms for the individual species, as given by equation 21 :

$$
R_{S}^{0}=\left\langle\left\langle R_{S}^{0}(\mathrm{~A}), R_{S}^{0}(\mathrm{~B})\right\rangle .\right.
$$

The second term is given by

$$
\begin{aligned}
R_{S}^{\text {leak }} & =-\frac{1}{3} \times \\
& \frac{\left\langle\left\langle R_{S}^{0}(\mathrm{~A})-R_{\Delta}^{0}(\mathrm{~A}), R_{S}^{0}(\mathrm{~B})-R_{\Delta}^{0}(\mathrm{~B})\right\rangle\right\rangle\left\langle\left\langle\Delta \Omega^{\mathrm{A}}, \Delta \Omega^{\mathrm{B}}\right\rangle^{2}\right.}{\left\langle\left\langle R_{S}^{0}(\mathrm{~A})-R_{\Delta}^{0}(\mathrm{~A}), R_{S}^{0}(\mathrm{~B})-R_{\Delta}^{0}(\mathrm{~B})\right\rangle^{2}+\omega_{J}^{2}\right.} .
\end{aligned}
$$

This term is due to the "leakage" of the singlet spin order operators into other terms, in particular $O_{\Delta}$, through the influence of the chemical shift frequency differences $\Delta \Omega^{\mathbb{X}}$, partially suppressed by $\omega_{J}^{3,6}$. This behaviour is captured by the inclusion of the term $R_{S}^{\text {leak }}$.

The third term is the most important one in the current context. It is given by

$$
R_{S}^{\mathrm{kin}}=\frac{1}{3} \frac{k_{\mathrm{BA}} k_{\mathrm{AB}}\left(\Delta \Omega^{\mathrm{A}}-\Delta \Omega^{\mathrm{B}}\right)^{2}}{\left(k_{\mathrm{BA}}+k_{\mathrm{AB}}\right)\left(\left(k_{\mathrm{BA}}+k_{\mathrm{AB}}\right)^{2}+\left\langle\left\langle\Delta \Omega^{\mathrm{A}}, \Delta \Omega^{\mathrm{B}}\right\rangle\right\rangle^{2}\right)} .
$$

This is called here the kinetic term since it depends on the kinetic rate constants $k_{\mathrm{AB}}$ and $k_{\mathrm{BA}}$, and cannot be expressed as a population-weighted average. Unlike the $R_{S}^{0}$ and $R_{S}^{\text {leak }}$ terms, the kinetic term $R_{S}^{\text {kin }}$ has no counterpart in the absence of chemical exchange. The term $R_{S}^{\text {kin }}$ expresses the direct influence of the chemical exchange process on the singlet relaxation, through the stochastic modulation of the chemical shift differences by the chemical process.

The treatment of singlet relaxation under ligand binding assumed that the singlet relaxation rate constant may be treated as a population-weighted average of the singlet relaxation rate constants of the individual species ${ }^{30-34}$, omitting the kinetic term. Although this assumption might have some merit under the conditions of ligand 
Table III. Numerical parameters for the two-site exchange model of the high-pH singlet relaxation of ${ }^{18} \mathrm{O}$-enriched 1,2${ }^{13} \mathrm{C}_{2}$ squarate. Species A corresponds to $\mathrm{SqH}^{-}$. Species B corresponds to $\mathrm{Sq}^{2-}$.

\begin{tabular}{l|l|l}
\hline$\Delta \delta^{\mathrm{A}}$ & $\delta_{\mathrm{csa}}^{\mathrm{A}}$ & $\beta_{P P}^{\mathrm{A}}$ \\
$49.67 \mathrm{ppb}$ & $80 \mathrm{ppm}$ & $3^{\circ}$ \\
\hline$\Delta \delta^{\mathrm{B}}$ & $\delta_{\mathrm{csa}}^{\mathrm{B}}$ & $\beta_{P P}^{\mathrm{B}}$ \\
$16.30 \mathrm{ppm}$ & $100 \mathrm{ppm}$ & $27^{\circ}$ \\
\hline \hline$b_{12} /(2 \pi)$ & $\xi_{\mathrm{erf}}(1-\kappa) /(2 \pi)^{2}$ & $\tau_{c}$ \\
$-2.76 \mathrm{kHz}$ & $0.015 \mathrm{mHz}$ & $100 \mathrm{ps}$ \\
\hline$k_{p}$ & $\mathrm{pKa}$ & \\
$10^{7} \mathrm{M}^{-1} \mathrm{~s}^{-1}$ & 3.4 &
\end{tabular}

binding, it is certainly not true in general. A more detailed discussion of such shortcomings will be given below.

\section{Application to $1,2-{ }^{13} \mathrm{C}$-squarate}

The proton exchange of squarate in solution is a complex problem since three chemical species, $\mathrm{Sq}^{2-}, \mathrm{SqH}^{-}$ and $\mathrm{SqH}_{2}$ are involved (see figure 3). Furthermore, each of the protonated $1,2-{ }^{13} \mathrm{C}_{2}$ species displays several isotopomers, depending on the position of the ${ }^{13} \mathrm{C}_{2}$ pair with respect to the protonation sites. For the sake of simplicity, we consider only the high-pH regime, ignoring the neutral $\mathrm{SqH}_{2}$ species, and neglecting the distinction between the multiple $1,2-{ }^{13} \mathrm{C}_{2}$ isotopomers of $\mathrm{SqH}^{-}$. Protonation (or deuteration) of squarate is taken into account solely through its influence on the ${ }^{13} \mathrm{C}$ chemical shifts. We ignore any direct effects of the proton or deuteron nuclear spins, through heteronuclear dipoledipole couplings or scalar couplings, and also ignore the effect of protonation on other interactions such as the ${ }^{13} \mathrm{C}-{ }^{13} \mathrm{C} J$-coupling. We also ignore the effect of ${ }^{18} \mathrm{O}$ or ${ }^{2} \mathrm{H}$ on the chemical equilibria. Although this model is highly simplified, we show below that it reproduces the main features of the observed data.

We consider the following chemical reaction

$$
\mathrm{SqH}^{-}+\mathrm{H}_{2} \mathrm{O} \underset{k_{p}}{\stackrel{k_{d}}{\rightleftharpoons}} \mathrm{Sq}^{2-}+\mathrm{H}_{3} \mathrm{O}^{+}
$$

characterised by the protonation second-order rate constant $k_{p}$ (in units of $\mathrm{M}^{-1} \mathrm{~s}^{-1}$ ), and the pseudo-first-order rate constant $k_{d}$ for the deprotonation (in units of $\mathrm{s}^{-1}$ ). The symbol $\mathrm{M}=$ mol $\mathrm{dm}^{-3}$ indicates molarity. In line with common practice, the solvent concentration is assumed to be effectively constant and factored into the deprotonation rate constant $k_{d}$.

For simplicity, we ignore the distinction between concentration and thermodynamic activity (this approximation is discussed further in section V.) We also assume

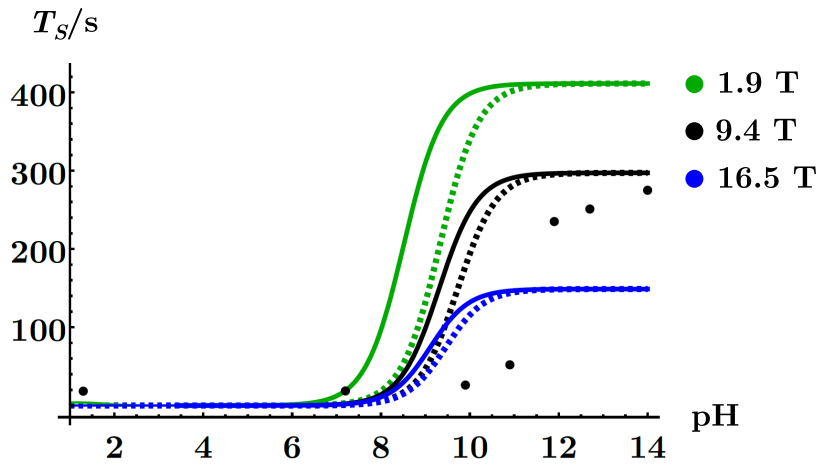

Figure 13. Two-site model predictions of the singlet relaxation time constant $T_{S}$ as a function of $\mathrm{pH}$ and magnetic field, using the parameters given in table III. Solid lines: the $T_{S}$ values are given by the inverse of the perturbative expression for $R_{S}$ (equation 23). Dotted lines: the $T_{S}$ values are given by the inverse of the approximate expression for $R_{S}$ (equations 47 and 48). Black circles correspond to experimentally acquired $T_{S}$ values at $9.4 \mathrm{~T}$ as shown in figure 2 .

that the base-to-acid kinetic rate constant $k_{\mathrm{AB}}$ is related to the protonation rate constant $k_{p}$ as follows:

$$
k_{\mathrm{AB}}=k_{p}\left[\mathrm{H}_{3} \mathrm{O}^{+}\right] \text {. }
$$

Neglecting again the distinction between activity and concentration, the concentration of hydronium ions depends on the $\mathrm{pH}$ value of the solution through

$$
\left[\mathrm{H}_{3} \mathrm{O}^{+}\right]=10^{-\mathrm{pH}} \times \mathrm{M}
$$

The exchange rate constant $k_{\mathrm{AB}}$ may therefore be expressed as follows:

$$
k_{\mathrm{AB}}=k_{p} 10^{-\mathrm{pH}} .
$$

The exchange rate constant $k_{\mathrm{BA}}$ is simply equal to the deprotonation rate constant $k_{d}$

$$
k_{\mathrm{BA}}=k_{d} \text {. }
$$

At the equivalence point $\mathrm{pH}=\mathrm{pK}_{a}$ the equilibrium concentrations of $\mathrm{Sq}^{2-}$ and $\mathrm{SqH}^{-}$are equal. We thus have

$$
\begin{aligned}
k_{d} & =k_{p} \frac{\left[\mathrm{Sq}^{2-}\right]}{\left[\mathrm{SqH}^{-}\right]}\left[\mathrm{H}_{3} \mathrm{O}^{+}\right] \\
& =k_{p}\left[\mathrm{H}_{3} \mathrm{O}^{+}\right] \\
& =k_{p} \times 10^{-\mathrm{pK}_{a}} .
\end{aligned}
$$

The required exchange rate constants are then given by

$$
\begin{aligned}
& k_{\mathrm{AB}}=k_{p} 10^{-\mathrm{pH}}, \\
& k_{\mathrm{BA}}=k_{p} 10^{-\mathrm{pK}_{a}} .
\end{aligned}
$$

Hence the kinetic rate constants $k_{\mathrm{AB}}$ and $k_{\mathrm{BA}}$ are solely characterised by the protonation rate constant $k_{p}$, the 
$\mathrm{pK}_{\mathrm{a}}$ value of the acid-base equilibrium, and the sample $\mathrm{pH}$. Since there is no available independent data on the protonation rate constant $k_{p}$, we treat this quantity as an adjustable parameter.

Equations 23 to 26 may be combined with equation 33 to provide an estimate for the decay rate constant $R_{S}$ of ${ }^{13} \mathrm{C}_{2}$ singlet order in ${ }^{18} \mathrm{O}$-enriched $1,2-{ }^{13} \mathrm{C}_{2}$ squarate, in the high-pH regime. The singlet relaxation time constant $T_{S}$ is given by the inverse of the rate constant, $T_{S}=R_{S}^{-1}$.

Evaluation of $T_{S}$ requires estimates of the isotropic shift differences $\Delta \Omega^{A}$ and $\Delta \Omega^{B}$, the ${ }^{13} \mathrm{C}$ chemical shift anisotropies and dipole-dipole coupling parameters, and the correlation time $\tau_{c}$ of the fluctuating interactions. The parameters used for the numerical modelling are given in table III. The $\mathrm{pK}_{\mathrm{a}}$ value was chosen to equal the known $\mathrm{pK}_{2}=3.4$ of squaric acid. The chemical shift differences and the CSA parameters are based on the computational chemistry estimates given in appendix A. The dipole-dipole coupling constant is based on the C-C bond length of $\sim 150 \mathrm{pm}$ for the squarate dianion ${ }^{94}$. The rotational correlation time $\tau_{C}=100$ ps agrees reasonably well with molecular dynamics simulations of the squarate dianion in aqueous solutions ${ }^{94}$. The minor influence of intermolecular interactions, including interactions with dissolved oxygen, is taken into account by a random-field contribution quantified by the parameter $\xi_{\text {erf }}(1-\kappa)$, recognizing that it is difficult to disentangle the correlation coefficient $\kappa$ from the mean-square fluctuation amplitude $\xi_{\text {erf }}$. The parameter $\xi_{\text {erf }}(1-\kappa)$ was chosen to match the experimentally observed $T_{S}$ values at $\mathrm{pH}=14$.

The solid curves in figure 13 shows analytical curves for $T_{S}=R_{S}^{-1}$ as a function of $\mathrm{pH}$ and magnetic field, using equation 23 for the rate constant, with parameters given in table III. The protonation rate constant $k_{p}=10^{7} \mathrm{M}^{-1} \mathrm{~s}^{-1}$ was chosen to obtain a rough qualitative match with experiment. This value is reasonably consistent with known protonation rate constants for carboxylic acids in solution, determined by NMR spectroscopy ${ }^{95,96}$ and fluorescence spectroscopy ${ }^{97,98}$.

Figure 13 also shows experimental values for $T_{S}$ at the magnetic field of $9.4 \mathrm{~T}$. Further values of $T_{S}$ at different magnetic fields are shown in figure 2. Although the correspondence between theory and experiment is qualitative at best, the salient features of the experimental data are reproduced. In particular the two-site model does reproduce the rapid transition from short $T_{S}$ to long $T_{S}$ at a $\mathrm{pH}$ value which greatly exceeds the $\mathrm{pK}_{\mathrm{a}}$ value of the acidbased equilibrium. The analytical curves also predict the decrease in the high-pH limit of $T_{S}$ with increasing magnetic field, which is due to the dominant chemical shift anisotropy relaxation in this regime.

The match between experiment and theory cannot be significantly improved within the framework of the twosite exchange model. In particular, adjustment of the protonation rate constant $k_{p}$ within reasonable limits does not lead to a significant improvement in the quality of the match. Possible reasons for the lack of a quantitative correspondence between experiment and theory are
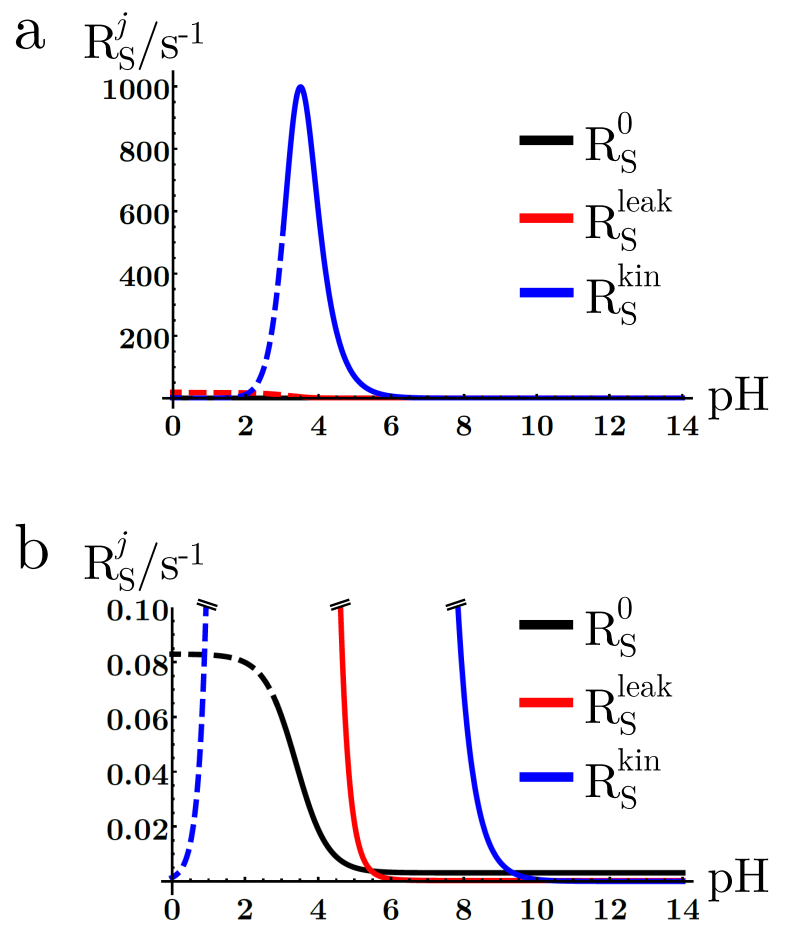

Figure 14. Individual contributions $R_{S}^{0}, R_{S}^{\text {leak }}$, and $R_{S}^{\mathrm{kin}}$, to the singlet relaxation rate constant $R_{S}$ (see equation 23) as a function of $\mathrm{pH}$, for the numerical parameter values given in table III. The vertical scale is greatly expanded in (b), as compared with (a). Double-struck lines indicate where the contributions $R_{S}^{\text {leak }}$ and $R_{S}^{\text {kin }}$ go off-scale. Dotted lines indicate the $\mathrm{pH}$ region in which the validity of the perturbative approximation to $R_{S}$ starts to decline.

discussed in section $\mathrm{V}$.

Figure 14 shows the individual contributions to the singlet relaxation rate constant $R_{S}$ as a function of $\mathrm{pH}$, using the parameters given in table III. The kinetic contribution is dominant for most of the relevant $\mathrm{pH}$ scale. It may be shown that the impact of the kinetic contribution is maximised for $\mathrm{pH}$ values around

$$
\mathrm{pH}_{\max }=\mathrm{pK}_{a}+\log _{10}(2) .
$$

For the current set of parameters this value corresponds to $\mathrm{pH}_{\max } \simeq 3.7$. The corresponding maximum is given by

$$
\left.R_{S}^{\mathrm{kin}}\right|_{\max } \simeq \frac{4}{27} 10^{\mathrm{pK}_{a}}\left(\Delta \Omega^{\mathrm{A}}\right)^{2} k_{p}^{-1},
$$

which from figure 14 corresponds roughly to $\left.R_{S}^{\text {kin }}\right|_{\max } \simeq$ $10^{4} \mathrm{~s}^{-1}$. This is 4 orders of magnitude larger than the corresponding background contribution $R_{S}^{0}$ at the same $\mathrm{pH}$ value! Clearly, the population weighted average $R_{S}$ is a very poor approximation to the singlet decay constant in this $\mathrm{pH}$ range.

For $\mathrm{pH}$ values $\mathrm{pH}>\mathrm{pH}_{\max }$ the kinetic contribution reduces in magnitude, and eventually becomes negligible. Nevertheless, figure $14 \mathrm{~b}$ shows that $R_{S}^{\mathrm{kin}}$ exceeds $R_{S}^{0}$ for 
$1.0 \lesssim \mathrm{pH} \lesssim 9.5$. So even for $\mathrm{pH}$ values 6 units above the $\mathrm{pK}_{\mathrm{a}}$ value, the populated-weighted average term $R_{S}^{0}$ is a poor approximation to the singlet decay rate constant.

Although the analysis above has been framed within the context of $1,2-{ }^{13} \mathrm{C}$ squarate, similar conclusions apply to other systems as well.

\section{E. Physical Interpretation}

The kinetic term $R_{S}^{\text {kin }}$ given by equation 26 is mainly responsible for the surprising singlet relaxation behaviour of $1,2-{ }^{13} \mathrm{C}_{2}$-squarate. In order to attach more physical meaning to $R_{S}^{\mathrm{kin}}$ consider an individual $\mathrm{Sq}^{2-}$ molecule in a high-pH solution. For high-pH solutions protonation events are rare. For example, at $\mathrm{pH}=10$, the protonation rate constant is estimated through equation 28 to be $k_{\mathrm{AB}} \simeq 1 \times 10^{-3} \mathrm{~s}^{-1}$. Hence, at $\mathrm{pH}=10$, a given $\mathrm{Sq}^{2-}$ molecule has to wait for about $1000 \mathrm{~s}$ before being protonated, on average.

From the protonation rate constant $k_{p}=10^{7} \mathrm{M}^{-1} \mathrm{~s}^{-1}$ and the $\mathrm{pK}_{\mathrm{a}}$ value of $\mathrm{pK}_{2}=3.4$, the deprotonation rate constant of $\mathrm{SqH}^{-}$is estimated through equation 32 to be given by $k_{d} \approx 4.0 \times 10^{3} \mathrm{~s}^{-1}$. This implies that the mean lifetime of a $\mathrm{SqH}^{-}$molecule, before losing its proton, is

$$
\tau_{\mathrm{SqH}^{-}}=k_{d}^{-1} \simeq 250 \mu \mathrm{s}
$$

at $\mathrm{pH}=10$. Hence, once a $\mathrm{Sq}^{2-}$ anion is protonated, the species $\mathrm{SqH}^{-}$persists in solution for around $250 \mu \mathrm{s}$ on average, before the proton is abstracted by the solvent again.

The lifetime of $\mathrm{SqH}^{-}$molecules in solution is statistically distributed. The probability $\delta p_{d}$ for an individual $\mathrm{SqH}^{-}$molecule to lose its proton during the small interval between time points $t=\tau$ and $t=\tau+\delta \tau$ is given by ${ }^{99}$

$$
\delta p_{d} \simeq k_{d} \delta \tau
$$

Hence the probability of a $\mathrm{SqH}^{-}$molecule keeping its proton during the same small time interval is given by

$$
1-\delta p_{d} \simeq 1-k_{d} \delta \tau
$$

where $k_{d}$ is the deprotonation rate constant. A finite interval $\tau$ may be subdivided into $n=\tau / \delta \tau$ small time intervals $\delta \tau$. Assuming statistical independence of events in successive time intervals (Markov process) ${ }^{100,101}$, the probability $p_{H}(\tau)$ for a molecule to remain protonated during the entire interval $\tau$ may be calculated as the product of the individual probabilities. In the limit of large $n$ the probability $p_{H}(\tau)$ becomes

$$
\begin{aligned}
p_{H}(\tau) & =\lim _{\delta \tau \rightarrow 0}\left(1-k_{d} \delta \tau\right)^{\tau / \delta \tau} \\
& =\exp \left(-k_{d} \tau\right)
\end{aligned}
$$

It follows that the probability of a molecule remaining protonated for the finite interval $\tau$, and then losing its proton during the following small time interval $[\tau, \tau+\delta \tau]$, is given by

$$
p_{H}(\tau) \delta p_{d} \simeq k_{d} \exp \left(-k_{d} \tau\right) \delta \tau
$$

Consider now a ${ }^{18} \mathrm{O}$-enriched $\quad 1,2-{ }^{13} \mathrm{C}_{2}$ squarate molecule in the singlet state:

$$
\left|S_{0}\right\rangle=2^{-1 / 2}(|\alpha \beta\rangle-|\beta \alpha\rangle)
$$

Since the isotropic shift difference between the ${ }^{13} \mathrm{C}$ sites (generated by the ${ }^{18} \mathrm{O}$ isotope shift) is very small, the singlet state is a good eigenstate of the coherent spin Hamiltonian. Hence a ${ }^{18} \mathrm{O}$-enriched $1,2-{ }^{13} \mathrm{C}_{2}$ squarate molecule in the nuclear singlet state remains in that state indefinitely - or more precisely, for times short compared to the relaxation time constants.

But now suppose that the singlet $\mathrm{Sq}^{2-}$ molecule is protonated at time $t=0$, giving a $\mathrm{SqH}^{-}$molecule. The change in electronic structure induces a chemical shift difference of $\Delta \delta$ between the ${ }^{13} \mathrm{C}$ sites, corresponding to a precession frequency difference of $\Delta \Omega=-\gamma B \Delta \delta$ in the magnetic field $B$. If the molecule remains in the protonated state for an interval $\tau$, the spin state of the ${ }^{13} \mathrm{C}_{2}$ pair state at time $t=\tau$ is given by

$$
\begin{aligned}
|\psi\rangle(\tau) & =2^{-1 / 2}\left(|\alpha \beta\rangle e^{-i \Delta \Omega \tau}-|\beta \alpha\rangle e^{i \Delta \Omega \tau}\right) \\
& =\left|S_{0}\right\rangle \cos \Delta \Omega \tau-i\left|T_{0}\right\rangle \sin \Delta \Omega \tau
\end{aligned}
$$

where the central triplet state is given by

$$
\left|T_{0}\right\rangle=2^{-1 / 2}(|\alpha \beta\rangle+|\beta \alpha\rangle)
$$

Since the state probabilities are given by the square magnitudes of the superposition coefficients, the probability of the ${ }^{13} \mathrm{C}_{2}$ pair being in the singlet state falls from unity to the value of $\cos ^{2} \Delta \Omega \tau$ at time $t=\tau$. Simultaneously the probability of the ${ }^{13} \mathrm{C}_{2}$ pair being in the central triplet state increases from zero to $\sin ^{2} \Delta \Omega \tau$ at time $t=\tau$.

Now consider a sub-ensemble of $\mathrm{Sq}^{2-}$ molecules, all protonated simultaneously at time $t=0$, and all in the nuclear singlet state. We may ask what the state of this sub-ensemble is at a later time $t=T$, where $T$ is long compared to the lifetime $\tau_{\mathrm{SqH}^{-}}$of a protonated squarate molecule, but sufficiently short that is unlikely that a squarate would become protonated during this time, and also short compared to the background relaxation rate constant $R_{S}^{0}$.

The probability that one of these $\mathrm{SqH}^{-}$molecules remains protonated for an interval $\tau$, and is then deprotonated during the following small interval $\delta \tau$, is given by $k_{d} \exp \left(-k_{d} \tau\right) \delta \tau$, through equation 40 . The probability that an individual $\mathrm{SqH}^{-}$molecule has that particular history, and is still in the singlet state after deprotonation, is obtained by multiplying that factor by $\cos ^{2} \Delta \Omega \tau$. The probability of any member of the sub-ensemble still being in the singlet state at the end of the interval $T$ is obtained by integrating over all possible values of the 
protonation duration $\tau$. This gives

$$
\begin{aligned}
p\left(\left|S_{0}\right\rangle \rightarrow\left|S_{0}\right\rangle\right) & =\int_{0}^{T} \cos ^{2} \Delta \Omega \tau k_{d} \exp \left(-k_{d} \tau\right) d \tau \\
& \simeq \int_{0}^{+\infty} \cos ^{2} \Delta \Omega \tau k_{d} \exp \left(-k_{d} \tau\right) d \tau \\
& \simeq \frac{k_{d}^{2}+2 \Delta \Omega^{2}}{k_{d}^{2}+4 \Delta \Omega^{2}} .
\end{aligned}
$$

The upper limit of the integral has been extended from $T$ to $+\infty$ by assuming that $T$ greatly exceeds the $\mathrm{SqH}^{-}$ lifetime.

Similarly the probability that the same sub-ensemble of squarate molecules, initially in the $\left|S_{0}\right\rangle$ state and protonated at time point $t=0$, are found in the central triplet state $\left|T_{0}\right\rangle$ after the interval $T$, is given by

$$
\begin{aligned}
p\left(\left|S_{0}\right\rangle \rightarrow\left|T_{0}\right\rangle\right) & =\int_{0}^{T} \sin ^{2} \Delta \Omega \tau k_{d} \exp \left(-k_{d} \tau\right) d \tau \\
& \simeq \int_{0}^{+\infty} \sin ^{2} \Delta \Omega \tau k_{d} \exp \left(-k_{d} \tau\right) d \tau \\
& \simeq \frac{2 \Delta \Omega^{2}}{k_{d}^{2}+4 \Delta \Omega^{2}} .
\end{aligned}
$$

The probabilities of transitions to the outer triplet states $\left|T_{ \pm 1}\right\rangle$ are negligible within the assumptions of this model.

Singlet order corresponds to a difference between the population of the singlet state and the mean population of the three triplet states. The change in ensemble singlet order due to the chemical and spin dynamical processes during a finite interval $T$ is therefore given by

$$
\begin{aligned}
\Delta_{\mathrm{SO}} & \simeq\left(p\left(\left|S_{0}\right\rangle \rightarrow\left|S_{0}\right\rangle\right)-\frac{1}{3} p\left(\left|S_{0}\right\rangle \rightarrow\left|T_{0}\right\rangle\right)\right)-1 \\
& \simeq-\frac{8 \Delta \Omega^{2}}{3\left(k_{d}^{2}+4 \Delta \Omega^{2}\right)} .
\end{aligned}
$$

The calculation above assumes that all molecules in the sub-ensemble are protonated at time point $t=0$. To calculate the contribution of the protonation-deprotonation events to the relaxation rate constant for singlet order, this quantity must be multiplied by the protonation rate constant $k_{\mathrm{AB}}$, given in equation 30 . This leads to the following estimate of the kinetic contribution to the relaxation of $\mathrm{Sq}^{2-}$ nuclear singlet order

$$
\begin{aligned}
R_{S}^{\text {kin }, \text { approx }} & \simeq-\Delta_{\mathrm{SO}} k_{\mathrm{AB}} \\
& =\frac{8 \Delta \Omega^{2}}{3\left(k_{d}^{2}+4 \Delta \Omega^{2}\right)} k_{p} 10^{-\mathrm{pH}}
\end{aligned}
$$

If the leakage term is ignored for simplicity, an approximate expression for the singlet relaxation rate constant is given by

$$
R_{S}^{\text {approx }} \simeq R_{S}^{0}+R_{S}^{\text {kin,approx }}
$$

where the "background" singlet relaxation rate constant is given by equation 24 , and the approximate form of the kinetic term is given by equation 47 .
Equation 47 has a straightforward physical interpretation. The factor $k_{p} 10^{-\mathrm{pH}}$ represents the rate constant for protonation of the $\mathrm{Sq}^{2-}$ anions. If the resulting $\mathrm{SqH}^{-}$ species has a small frequency difference between the two ${ }^{13} \mathrm{C}$ sites $(\Delta \Omega \rightarrow 0)$, the singlet state remains a good energy eigenstate in the $\mathrm{SqH}^{-}$species and there is no significant evolution of the singlet order before the molecule loses its proton again. The kinetic contribution to $R_{S}$ becomes very small in this limit, so that $R_{S}$ is dominated by the term $R_{S}^{0}$, which is the population-weighted average of the singlet relaxation rate constants in the individual species. This is the approach used previously for the studies of ligand binding by singlet $\mathrm{NMR}^{30-34}$.

If the chemical shift frequency difference in the $\mathrm{SqH}^{-}$ species is large $\left(|2 \Delta \Omega| \gg k_{d}\right)$, the first term in equation 47 approaches $2 / 3$, and the kinetic contribution to $R_{S}$ acquires a very simple form:

$$
R_{S}^{\mathrm{kin}, \text { approx }} \rightarrow \frac{2}{3} k_{p} 10^{-\mathrm{pH}}
$$

In this limit, every protonation event destroys $2 / 3$ of the singlet order, so that the kinetic contribution to $R_{S}$ is simply equal to the protonation rate constant of $\mathrm{Sq}^{2-}$ anions in solution, multiplied by $2 / 3$. Note that equation 49 is completely free of spin system parameters except for their role in setting the limits of the relevant approximations.

For the system considered here, the factor $\Delta_{\mathrm{SO}}$ is given by $\sim 0.64$ in a magnetic field of $9.4 \mathrm{~T}$, i.e. slightly less than the $2 / 3$ limit. Under these conditions, every protonation event destroys on average about $64 \%$ of the nuclear singlet order of a $\mathrm{Sq}^{2-}$ anion, and the kinetic contribution to $R_{S}$ is equal to about $64 \%$ of the protonation rate constant of squarate ions in solution.

Figure 13 compares $\mathrm{pH}$-dependent $T_{S}$ values derived by the perturbative expression for $R_{S}$ (equation 23) with those given by the simplified approach (equations 47 and 48), as a function of magnetic field. The reasonable agreement between these two forms of analysis validates the approximations made in the simplified model, in the case of a sufficiently large shift difference in the protonated forms.

The physical model described above is readily extended, in the high-pH limit, to the case where the $\mathrm{Sq}^{2-}$ is in chemical exchange with multiple inequivalent forms of the $\mathrm{SqH}^{-}$species. In the current case, there are four inequivalent forms of $1,2-{ }^{13} \mathrm{C}_{2}-\mathrm{SqH}^{-}$, since the proton may be attached to any one of the four oxygen atoms, leading in each case to different chemical shift changes for the two adjacent ${ }^{13} \mathrm{C}$ sites (see appendix $\mathrm{A}$ ). The result of this analysis is that equation 47 should be replaced by

$$
R_{S}^{\mathrm{kin}, \text { approx }} \simeq \sum_{i=1}^{4} \frac{8 \Delta \Omega_{i}^{2}}{3\left(k_{d}^{2}+4 \Delta \Omega_{i}^{2}\right)} k_{p} 10^{-\mathrm{pH}},
$$

where $\Delta \Omega_{i}$ denotes the difference in the ${ }^{13} \mathrm{C}$ shift frequencies for the $i$ th $1,2-{ }^{13} \mathrm{C}-\mathrm{SqH}^{-}$species. This substitution makes little difference to the computed curves, except for 
an effective increase in the protonation rate constant $k_{p}$, which is to be expected since there are four protonation sites in this multi-site model, instead of just one. Since $k_{p}$ is an adjustable parameter anyway in our theory, the multi-site protonation model does not lead to a significant improvement in the agreement between theory and simulation.

\section{v. DISCUSSION}

The study of ${ }^{13} \mathrm{C}$ nuclear singlet relaxation in ${ }^{18} \mathrm{O}$ enriched $1,2-{ }^{13} \mathrm{C}_{2}$ squarate reveals a surprising dependence of the singlet relaxation time constant $T_{S}$ on the $\mathrm{pH}$ of the solution. A rather sharp transition is observed, from a short $T_{S}$ at $\mathrm{pH}$ values below $\sim 10$, to long $T_{S}$ values above $\mathrm{pH} \sim 12$. The position of this transition is more than $6 \mathrm{pH}$ points above the known $\mathrm{pK}_{\mathrm{a}}$ values of squaric acid.

A previously described model of nuclear singlet relaxation in exchanging systems, developed for ligand binding studies, assumed that the singlet relaxation rate constant is given by a population-weighted average of the relaxation rate constants for the exchanging species ${ }^{30-34}$. That model cannot explain the squaric acid observations, since the equilibrium populations of the protonated squarate species are negligible in the $\mathrm{pH}$ range of interest.

In this paper we show theoretically that there is an additional contribution to the singlet relaxation rate constant in chemically exchanging systems, which we call the kinetic term. This term cannot be described as a thermodynamic average over equilibrium concentrations. The kinetic term depends on kinetic rate constants and can be very significant for fast reactions such as protonation in solution.

The kinetic contribution to $R_{S}$ is derived for a two-site exchange model, under various approximations, and is shown to reproduce the main features of the experimental data - especially the position of the transition in singlet relaxation behaviour, which is at a $\mathrm{pH}$ value which is far displaced from the acid/base equilibrium point.

These observations and analysis indicate that nuclear singlet relaxation may be highly sensitive to chemical exchange, even in cases where the equilibrium overwhelmingly favours one of the exchanging species. In the current case, the concentration of the $\mathrm{Sq}^{2-}$ anion exceeds that of $\mathrm{SqH}^{-}$anion by a factor of more than $10^{6}$ at $\mathrm{pH}$ $=10$. Nevertheless, the rare and transient $\mathrm{SqH}^{-}$species has a very significant effect on ${ }^{13} \mathrm{C}_{2}$ singlet relaxation.

This effect presents both difficulties and opportunities. The sensitivity of nuclear singlet relaxation to rare protonation events is likely to make it even more difficult to utilize long-lived states for the storage and transport of hyperpolarized spin order, especially in protic solvents. It is probably no coincidence that the longest observed singlet lifetimes have been observed in aprotic solvents ${ }^{12}$, including liquid carbon dioxide ${ }^{19}$. It is likely that this effect has already contributed to some observations in the literature, one example being the relatively rapid decay of ${ }^{13} \mathrm{C}_{2}$ singlet order in ${ }^{18} \mathrm{O}$-labelled oxalate ${ }^{76}$.

On the other hand, the extreme sensitivity of nuclear singlet relaxation to rare chemical events suggest applications of this phenomenon to the determination of chemical rate constants in dynamic equilibria, under conditions beyond the range of other techniques. As shown by equation 49 , nuclear singlet relaxation may provide a direct measurement of small kinetic rate constants. Applications to the study of conformational equilibria in biomolecules, such as the study of minor or "unobservable" species, are conceivable ${ }^{102-108}$.

The theory given here may also be of value for other phenomena involving a combination of chemical and spin dynamics, such as Signal Amplification by Reversible Exchange (SABRE) 109-111, Chemically Induced Dynamic Nuclear Polarization (CIDNP) ${ }^{112,113}$, and spin-isomer conversion of dissolved $\mathrm{H}_{2}$ gas ${ }^{114}$.

Although the theory given here reproduces well the main features of the experimental observations, precise agreement between theory and experiment remains elusive. As shown in figure 13, the transition between the long and short $T_{S}$ regimes occurs at a $\mathrm{pH}$ value of around 11 , whereas the theory predicts that the transition should occur at a pH of around 9.5. Although the relaxation theory relies on several assumptions and approximations, our current view is that the central core of the theory is well-grounded and that major theoretical errors may safely be excluded. Note that two very different versions of the relaxation theory reach similar conclusions, as also shown in figure 13. The deviations between these two forms of the theory are well-understood since the theory leading to the approximate form of equation 47 employs several approximations that do not apply to the more accurate perturbative expression in equation 26 . We have also taken precautions to exclude errors in the experimental measurements including $\mathrm{pH}$ calibration or drift issues or perturbations due to isotope effects, as described in section II A. We therefore conclude that the remaining deviations between the experimental results and the relaxation theory are not due to problems with the relaxation theory or the experiment, but are due to the behaviour of the system itself.

The predicted $\mathrm{pH}$ dependence of the singlet relaxation rate constant $R_{S}$ relies on the validity of equation 30, which describes the $\mathrm{pH}$-dependence of the baseto-acid conversion rate constant $k_{\mathrm{AB}}$. This relationship is suspect, for several reasons. Firstly, this equation ignores the difference between concentrations and thermodynamic activities, which are only equivalent in very dilute solutions. Secondly, it is known that the kinetic rate constants for reactions between charged species depend on ionic strength ${ }^{115}$.

In our experiments, the ionic strength of the solution increases from about $0.1 \mathrm{M}$ to about $0.3 \mathrm{M}$ as the $\mathrm{pH}$ is increased by addition of sodium hydroxide. This change in ionic strength with increase of $\mathrm{pH}$ is likely to perturb the $\mathrm{SqH}^{-} \rightleftharpoons \mathrm{Sq}^{2-}+\mathrm{H}^{+}$equilibrium in favour of the 
$\mathrm{SqH}^{-}$species. However, at the time of writing, it is not clear whether this effect is large enough to account for the observed discrepancy between the experimental measurements and theoretical curves, shown prominently in figure 13. The possibility remains open that these experiments probe very slow protonation phenomena which do not behave according to standard kinetic theories, and which have so far escaped detailed scrutiny. Further work is needed to quantify the role of ionic strength and to test this speculative hypothesis.

In summary, this study has shown that in suitable circumstances, very slow protonation rate constants may be studied quantitatively by singlet NMR. We are not aware of other physical methods capable of detecting such infrequent chemical events at equilibrium. The challenges of interpretation appear not to be due to problems with the underlying theory or the measurements themselves, but to the reconciliation of the estimated rate constants with theories of chemical equilibria.

\section{CONFLICTS OF INTEREST}

There are no conflicts to declare.

\section{ACKNOWLEDGMENTS}

This research was funded by the European Research Council (786707-FunMagResBeacons), and the Engineering and Physical Sciences Research Council (grants EP/P009980/1 and EP/P030491/1). L.D. acknowledges support from the Marie Skłodowska-Curie program of the European Union (grant number 766402). A.K. acknowledges support from the Russian Ministry of Education and Science (contract number 075-15-2021-580). We thank Weidong Gong for instrumental help, and Phil Bartlett and Andrea Russell for advice on chemical equilibria.

\section{DATA AVAILABILITY STATEMENT}

The data that support the findings of this study are available from the corresponding author upon reasonable request.

\section{Appendix A: Shielding tensor calculations}

Estimates of the ${ }^{13} \mathrm{C}$ nuclear shielding tensors for $\mathrm{SqH}^{-}$ and $\mathrm{Sq}^{2-}$, were obtained by the SCF-GIAO algorithm as implemented in Gaussian ${ }^{119}$. All calculations assumed a water solvation model and neglect secondary isotope shifts.

Since the $\mathrm{Sq}^{2-}$ molecule has a high symmetry, the principal axes of the symmetric parts of each shielding tensor are all precisely aligned with a molecular symmetry axis.

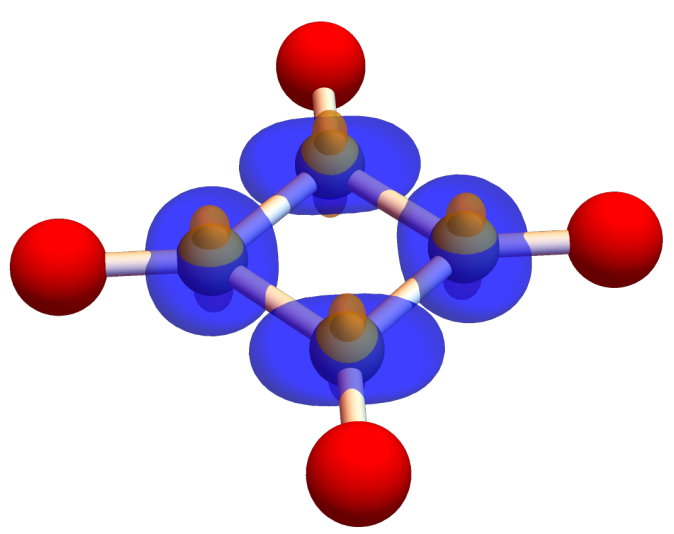

Figure 15. Calculated shielding tensors for the four carbon sites of $\mathrm{Sq}^{2-}$. The symmetric parts of the shielding tensors are represented by ovaloids ${ }^{116,117}$, generated by the Ovaloid symbol of SpinDynamica software ${ }^{118}$. Blue and orange surfaces indicate opposite signs of the shielding. The carbon and oxygen atoms are represented by grey and red spheres, respectively.

The principal values of all four ${ }^{13} \mathrm{C}$ shielding tensors are the same and are calculated to be $\sigma_{X X}=-68.6 \mathrm{ppm}$, $\sigma_{Y Y}=-97.4 \mathrm{ppm}, \sigma_{Z Z}=+74.7 \mathrm{ppm}$. For each ${ }^{13} \mathrm{C}$ site, the $\mathrm{X}$ principal axis is parallel to the line joining that site with the centre of the square, the $\mathrm{Z}$ principal axis is perpendicular to the plane of the square, and the $\mathrm{Y}$ axis is perpendicular to both $\mathrm{X}$ and $\mathrm{Y}$. Figure 15 depicts the calculated shielded tensors as blue and orange ovaloid surfaces ${ }^{116,117}$ superposed on the $\mathrm{Sq}^{2-}$ molecular structure.

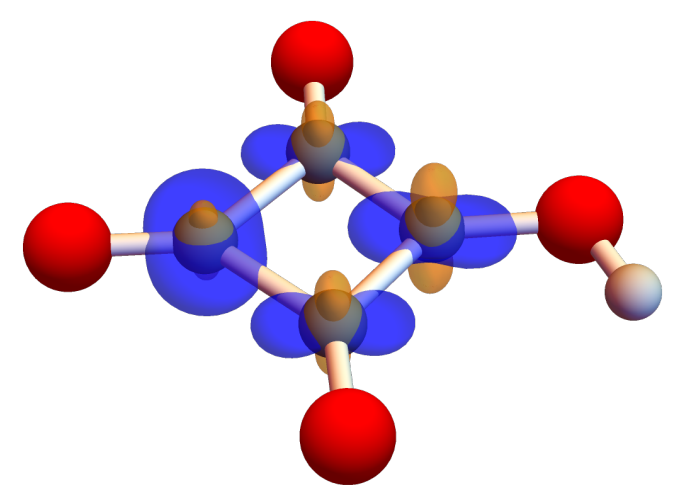

Figure 16. Calculated shielding tensors for the four carbon sites of $\mathrm{SqH}^{-}$. The symmetric parts of the shielding tensors are represented by ovaloids ${ }^{116,117}$. Note the strong perturbation of the shielding tensors for the three ${ }^{13} \mathrm{C}$ sites closest to the protonation site, relative to $\mathrm{Sq}^{2-}$ (see figure 15). 
The energy-minimized structure of the $\mathrm{SqH}^{-}$molecule has low symmetry, as shown in figure 16. Assume that the proton is attached to oxygen O1, which is bound to carbon $\mathrm{C} 1$. Define an axis system such that the $x$-axis is parallel to the line joining $\mathrm{C} 1$ to the centre of the square, the $z$-axis is perpendicular to the square, and the $y$-axis is perpendicular to the other two axes. The shielding tensors for the four carbon sites, expressed in this axis system, are as follows:

$$
\begin{aligned}
\sigma_{1}\left(\mathrm{SqH}^{-}\right) & =\left(\begin{array}{ccc}
-94.6 & 9.5 & 0 . \\
3.1 & -48.8 & 0 . \\
0 . & 0 . & 95.8
\end{array}\right) \mathrm{ppm}, \\
\sigma_{2}\left(\mathrm{SqH}^{-}\right) & =\left(\begin{array}{ccc}
-90.1 & -13.1 & 0 . \\
16.1 & -24.2 & 0 . \\
0 . & 0 . & 74.1
\end{array}\right) \mathrm{ppm}, \\
\sigma_{3}\left(\mathrm{SqH}^{-}\right) & =\left(\begin{array}{ccc}
-67.6 & 0.5 & 0 . \\
3.8 & -103.8 & 0 . \\
0 . & 0 . & 61.3 \\
0 . & 17.9 & 0 . \\
-89.4 & 10.1 \\
-14.1 & -29.3 & 0 . \\
0 . & 0 . & 76.2
\end{array}\right) \mathrm{ppm}, \\
\sigma_{4}\left(\mathrm{SqH}^{-}\right) & \text {ppm. }
\end{aligned}
$$

The symmetric parts of these tensors are depicted by the ovaloid surfaces in figure 16 . The asymmetric disposition of the hydrogen atom breaks the symmetry; the numbering of the atoms is such that the $\mathrm{H}$ atom is closer to $\mathrm{O} 4$ than to $\mathrm{O} 2$.

The calculated values of the isotropic parts of the shielding tensors are given in table $\mathrm{A}$. The quantity $\left\langle\sigma^{\text {iso }}\right\rangle$ is the average of the isotropic ${ }^{13} \mathrm{C}$ shielding factors over all four positions. Since the shielding $\sigma$ and chemical shift $\delta$ have opposite signs, the increase in mean isotropic shielding when $\mathrm{Sq}^{2-}$ is protonated corresponds roughly to the decrease in the isotropic chemical shift $\delta_{\text {iso }}$ when the $\mathrm{pH}$ is decreased (see figure 7).

The quantity $\left\langle\left|\Delta \sigma^{\text {iso }}\right|\right\rangle$ in table $\mathrm{A}$ is the mean difference in isotropic shielding factor between adjacent ${ }^{13} \mathrm{C}$ sites, neglecting the sign. This quantity corresponds roughly to the parameter $\Delta \delta$ used in the two-site calculations (table III).

\section{Appendix B: Detailed Relaxation Theory}

\section{Combined matrix representations}

Any site-specific density operators may be expanded as a superposition of orthonormal spin operators

$$
\rho^{\mathbb{X}}(t)=\sum_{n} q_{n}^{\mathbb{X}}(t) Q_{n}^{\mathbb{X}}
$$

\begin{tabular}{l|l|l} 
& $\mathrm{Sq}^{2-}$ & $\mathrm{SqH}^{-}$ \\
\hline$\sigma_{1}^{\text {iso }}$ & $-30.5 \mathrm{ppm}$ & $-15.9 \mathrm{ppm}$ \\
$\sigma_{2}^{\text {iso }}$ & $-30.5 \mathrm{ppm}$ & $-13.4 \mathrm{ppm}$ \\
$\sigma_{3}^{\text {iso }}$ & $-30.5 \mathrm{ppm}$ & $-36.7 \mathrm{ppm}$ \\
$\sigma_{4}^{\text {iso }}$ & $-30.5 \mathrm{ppm}$ & $-14.2 \mathrm{ppm}$ \\
\hline$\left\langle\sigma^{\text {iso }}\right\rangle$ & $-30.5 \mathrm{ppm}$ & $-20.0 \mathrm{ppm}$ \\
$\Delta \sigma_{\mathrm{rms}}^{\text {iso }}$ & 0 & $16.3 \mathrm{ppm}$
\end{tabular}

Table IV. Quantum chemistry estimates for the isotropic shielding parameters for the ${ }^{13} \mathrm{C}$ sites in $\mathrm{Sq}^{2-}$ and $\mathrm{SqH}^{-}$, assuming a water solvation model. The sites are numbered so that the hydrogen is attached to $\mathrm{O} 1$ and is closer to $\mathrm{O} 4$ than to O2. The row labelled $\left\langle\sigma^{\text {iso }}\right\rangle$ gives the average isotopic shielding of all four ${ }^{13} \mathrm{C}$ positions. The row labelled $\Delta \sigma_{\mathrm{rms}}^{\text {iso }}$ gives the root-mean-square difference in isotropic shielding factors between adjacent ${ }^{13} \mathrm{C}$ positions.

The coefficients $q_{n}(t)$ may be derived from the trace inner product

$$
q_{n}^{\mathbb{X}}(t)=\operatorname{Tr}\left\{\left(Q_{n}^{\mathbb{X}}\right)^{\dagger} \rho^{\mathbb{X}}(t)\right\}
$$

In general there are $N_{\mathbb{L}}=N_{\mathbb{H}}^{2}$ coefficients for each site, where $N_{\mathbb{H}}$ is the dimension of the corresponding Hilbert space. The site-specific density operator is then fully characterised by the vector

$$
\mathbf{q}^{\mathbb{X}}(t)=\left[q_{1}^{\mathbb{X}}(t), \ldots, q_{N_{\mathbb{L}}}^{\mathbb{X}}(t)\right],
$$

where boldfaced letters are used to emphasize an explicit matrix representation of any object.

The matrix representation for a site-specific Liouvillian is defined via

$$
\mathbf{L}_{m n}^{\mathbb{X}}=\operatorname{Tr}\left\{\left(Q_{m}^{\mathbb{X}}\right)^{\dagger} \hat{L}^{\mathbb{X}} Q_{n}^{\mathbb{X}}\right\} .
$$

Following equation 10 the state of the combined ensemble may be obtained by "stacking" the vectors $\mathbf{q}^{\mathrm{A}}(t)$ and $\mathbf{q}^{\mathrm{B}}(t)$ on top of each other

$$
\mathbf{q}(t)=\left[q_{1}^{\mathrm{A}}(t), \ldots, q_{N_{\mathbb{L}}}^{\mathrm{A}}(t), q_{1}^{\mathrm{B}}(t), \ldots, q_{N_{\mathbb{L}}}^{\mathrm{B}}(t)\right] .
$$

The equation of motion for $\mathbf{q}(t)$ is then given by

$$
\frac{d}{d t} \mathbf{q}(t)=\mathbf{G} \mathbf{q}(t)=(\mathbf{L}+\mathbf{K}) \mathbf{q}(t),
$$

with

$$
\begin{aligned}
& \mathbf{L}=\mathbf{L}^{\mathrm{A}} \oplus \mathbf{L}^{\mathrm{B}}, \\
& \mathbf{K}=\left[\begin{array}{rr}
-k_{\mathrm{BA}} & k_{\mathrm{AB}} \\
k_{\mathrm{BA}} & -k_{\mathrm{AB}}
\end{array}\right] \otimes \mathbb{1}_{N_{\mathbb{L}}} .
\end{aligned}
$$

Here, $(\oplus)$ represents a matrix direct sum, $(\otimes)$ a matrix direct product and $\mathbb{1}_{N_{\mathbb{L}}}$ a unity matrix of dimension $N_{\mathbb{L}}$. 


\section{Perturbative treatment}

The generator $\hat{G}$ or its representation $\mathbf{G}$ may be characterised in terms of its eigenvalues and eigenvectors

$$
\mathbf{G v}_{j}=\Lambda_{j} \mathbf{v}_{j}
$$

The eigenvalues $\Lambda_{j}$ are in general complex

$$
\Lambda_{j}=+i \omega_{j}-\lambda_{j}
$$

with $\omega_{j}$ characterising the oscillatory behaviour and $\lambda_{j} \geq 0$ characterising the decaying behaviour of the eigenvector $\mathbf{v}_{j}$.

The evolution of any initial density operator $\mathbf{q}(0)$ may then be expressed as follows

$$
\mathbf{q}(t)=\exp \{\mathbf{G} t\} \mathbf{q}(0)=\sum_{j} c_{j}(0) \exp \left\{\Lambda_{j} t\right\} \mathbf{v}_{j}
$$

The coefficients $c_{j}(0)$ represent the projections of $\mathbf{q}(0)$ along the corresponding eigenvector $\mathbf{v}_{j}$ at $t=0$.

In general, obtaining all eigenvalues and eigenoperators of $\mathbf{G}$ is difficult, and one typically has to resort to an approximate treatment of the problem. In general however the exchange rates $k_{\mathrm{BA}}$ and $k_{\mathrm{AB}}$ may be highly asymmetric, which poses a problem for typical approximation techniques. But we may symmetrise the exchange matrix $\mathbf{K}$ via a similarity transformation $\mathbf{V}$

$$
\mathbf{V}=\left[\begin{array}{cc}
\sqrt{k_{\mathrm{AB}}} & 0 \\
0 & \sqrt{k_{\mathrm{BA}}}
\end{array}\right],
$$

$$
\begin{aligned}
\tilde{\mathbf{K}}=\mathbf{V}^{-1} \mathbf{K} \mathbf{V} & =\left[\begin{array}{cc}
-k_{\mathrm{BA}} & \sqrt{k_{\mathrm{AB}} k_{\mathrm{BA}}} \\
\sqrt{k_{\mathrm{AB}} k_{\mathrm{BA}}} & -k_{\mathrm{AB}}
\end{array}\right] . \\
& =\left[\begin{array}{cc}
-k_{\mathrm{BA}} & w \\
w & -k_{\mathrm{AB}}
\end{array}\right],
\end{aligned}
$$

where $w$ represents the geometric mean of the forward and backward exchange rates ${ }^{120}$.

The transformed generator of motion then takes the form

$$
\tilde{\mathbf{G}}=\left(\mathbf{V}^{-1} \otimes \mathbb{1}_{N_{\mathbb{L}}}\right) \mathbf{G}\left(\mathbf{V} \otimes \mathbb{1}_{N_{\mathbb{L}}}\right)=\mathbf{L}+\tilde{\mathbf{K}}
$$

It is easy to see that $\tilde{\mathbf{G}}$ and $\mathbf{G}$ have the same eigenvalues. We therefore omit the tilde from now on, to keep the notation somewhat compact.

A SpinDynamica ${ }^{118}$ calculation shows that the relevant subspace of $\mathbf{G}$ is spanned by the following ten operators

$$
\begin{aligned}
& \mathcal{B}=\left\{b_{1}, b_{2}, b_{3}, b_{4}, b_{5}, b_{6}, b_{7}, b_{8}, b_{9}, b_{10}\right\} \\
&=\left\{1 / \sqrt{3}\left(2 I_{1 z}^{\mathrm{A}} I_{2 z}^{\mathrm{A}}+I_{1-}^{\mathrm{A}} I_{2+}^{\mathrm{A}}+I_{1+}^{\mathrm{A}} I_{2-}^{\mathrm{A}}\right),\right. \\
& 1 / \sqrt{6}\left(4 I_{1 z}^{\mathrm{A}} I_{2 z}^{\mathrm{A}}-I_{1-}^{\mathrm{A}} I_{2+}^{\mathrm{A}}-I_{1+}^{\mathrm{A}} I_{2-}^{\mathrm{A}}\right), \\
& 1 / \sqrt{2}\left(I_{1 z}^{\mathrm{A}}+I_{2 z}^{\mathrm{A}}\right), \\
& 1 / 2\left(I_{2 z}^{\mathrm{A}}-I_{1 z}^{\mathrm{A}}+I_{1-}^{\mathrm{A}} I_{2+}^{\mathrm{A}}-I_{1+}^{\mathrm{A}} I_{2-}^{\mathrm{A}}\right) \\
& 1 / 2\left(I_{2 z}^{\mathrm{A}}-I_{1 z}^{\mathrm{A}}-I_{1-}^{\mathrm{A}} I_{2+}^{\mathrm{A}}+I_{1+}^{\mathrm{A}} I_{2-}^{\mathrm{A}}\right) \\
& 1 / \sqrt{3}\left(2 I_{1 z}^{\mathrm{B}} I_{2 z}^{\mathrm{B}}+I_{1-}^{\mathrm{B}} I_{2+}^{\mathrm{B}}+I_{1+}^{\mathrm{B}} I_{2-}^{\mathrm{B}}\right), \\
& 1 / \sqrt{6}\left(4 I_{1 z}^{\mathrm{B}} I_{2 z}^{\mathrm{B}}-I_{1-}^{\mathrm{B}} I_{2+}^{\mathrm{B}}-I_{1+}^{\mathrm{B}} I_{2-}^{\mathrm{B}}\right), \\
& 1 / \sqrt{2}\left(I_{1 z}^{\mathrm{B}}+I_{2 z}^{\mathrm{B}}\right), \\
& 1 / 2\left(I_{2 z}^{\mathrm{B}}-I_{1 z}^{\mathrm{B}}+I_{1-}^{\mathrm{B}} I_{2+}^{\mathrm{B}}-I_{1+}^{\mathrm{B}} I_{2-}^{\mathrm{B}}\right) \\
&\left.1 / 2\left(I_{2 z}^{\mathrm{B}}-I_{1 z}^{\mathrm{B}}-I_{1-}^{\mathrm{B}} I_{2+}^{\mathrm{B}}+I_{1+}^{\mathrm{B}} I_{2-}^{\mathrm{B}}\right)\right\} .
\end{aligned}
$$

Out of these ten operators there is a sufficient spectral separation of the operators $\left(b_{1}, b_{5}, b_{6}, b_{10}\right)$ from the remaining operators to isolate the corresponding $4 \times 4$ sub-matrix, which we denote by $\mathbf{g}$

$$
\mathbf{g}=\left[\begin{array}{cccc}
-k_{\mathrm{AB}}-R_{S}^{0}(\mathrm{~A}) & i 3^{-1 / 2} \Delta \Omega^{\mathrm{A}} & \sqrt{k_{\mathrm{AB}} k_{\mathrm{BA}}} & 0 \\
i 3^{-1 / 2} \Delta \Omega^{\mathrm{A}} & -k_{\mathrm{AB}}-R_{\Delta}^{0}(\mathrm{~A})+i \omega_{J} & 0 & \sqrt{k_{\mathrm{AB}} k_{\mathrm{BA}}} \\
\sqrt{k_{\mathrm{AB}} k_{\mathrm{BA}}} & 0 & -k_{\mathrm{BA}}-R_{S}^{0}(\mathrm{~B}) & i 3^{-1 / 2} \Delta \Omega^{\mathrm{B}} \\
0 & \sqrt{k_{\mathrm{AB}} k_{\mathrm{BA}}} & i 3^{-1 / 2} \Delta \Omega^{\mathrm{B}} & -k_{\mathrm{BA}}-R_{\Delta}^{0}(\mathrm{~B})+i \omega_{J}
\end{array}\right] .
$$

The diagonal relaxation rates are defined as follows

$R_{S}^{\mathbb{X}}=$

$4(1-\kappa) \xi_{\mathrm{erf}}+\frac{10}{9}\left(\xi_{\mathrm{csa}}^{1}(\mathbb{X})+\xi_{\mathrm{csa}}^{2}(\mathbb{X})-2 \xi_{\mathrm{csa}}^{12}(\mathbb{X}) d_{00}^{2}\left(\beta_{\mathbb{X}}\right)\right)$,

$R_{\Delta}^{0}(\mathrm{~B})=$

$\left.(3-\kappa) \xi_{\mathrm{erf}}+\frac{5}{12} \xi_{\mathrm{dd}}+\frac{5}{6}\left(\xi_{\mathrm{csa}}^{1}+\xi_{\mathrm{csa}}^{2}\right)-\frac{2}{3} \xi_{\mathrm{csa}}^{12}(\mathbb{X}) d_{00}^{2}\left(\beta_{\mathbb{X}}\right)\right)$
The operators $\left(b_{1}, b_{6}\right)$ represent singlet order for sites $\mathrm{A}$ and $\mathrm{B}$, whereas the operators $\left(b_{5}, b_{10}\right)$ represent anti- 
symmetric spherical tensors operators of rank $k=1$ for sites $\mathrm{A}$ and $\mathrm{B}$. Motivated by this observation we introduce a rotation matrix $\mathbf{R}(\theta, \phi)$ mixing states $\left(b_{1}, b_{6}\right)$ and $\left(b_{5}, b_{10}\right)$

$$
\mathbf{R}(\theta, \phi)=\left[\begin{array}{cccc}
\cos (\theta) & 0 & -\sin (\theta) & 0 \\
0 & \cos (\phi) & 0 & -\sin (\phi) \\
\sin (\theta) & 0 & \cos (\theta) & 0 \\
0 & \sin (\phi) & 0 & \cos (\phi)
\end{array}\right]
$$

and define two exchange rate dependent mixing angles

$$
\begin{aligned}
\theta_{\mathrm{mix}}^{1} & =\frac{1}{2} \arctan 2\left(\mathbf{g}_{11}-\mathbf{g}_{33}, 2 \mathbf{g}_{13}\right) \\
& =\frac{1}{2} \arctan 2\left(k_{\mathrm{AB}}-k_{\mathrm{BA}}-R_{S}^{0}(\mathrm{~A})+R_{S}^{0}(\mathrm{~B}), 2 w\right), \\
\theta_{\mathrm{mix}}^{2} & =\frac{1}{2} \arctan 2\left(\mathbf{g}_{22}-\mathbf{g}_{44}, 2 \mathbf{g}_{24}\right) \\
& =\frac{1}{2} \arctan 2\left(k_{\mathrm{AB}}-k_{\mathrm{BA}}-R_{\Delta}^{0}(\mathrm{~A})+R_{\Delta}^{0}(\mathrm{~B}), 2 w\right) .
\end{aligned}
$$

Here, it is necessary to use the arctan2 function since the difference $k_{\mathrm{AB}}-k_{\mathrm{BA}}$ may either be positive or negative.

The mixing angle $\theta_{\text {mix }}^{1}$ describes the exchange induced mixing of singlet order for sites $\mathrm{A}$ and $\mathrm{B}$, whereas $\theta_{\text {mix }}^{2}$ describes the exchange induced mixing of the corresponding anti-symmetric terms. Although slightly unusual at first, this is nothing surprising. In the intermediate exchange regime it is impossible to strictly separate the dynamical features of spins in site A and B. This implies that the eigenvectors (eigenoperators) of $\mathbf{G}$ are also inseparable.

The rotation matrix $\mathbf{R}\left(\theta_{\text {mix }}^{1}, \theta_{\text {mix }}^{2}\right)$ may be used to transform $\mathbf{g}$ into a tilted basis. The transformed matrix representation takes the generic form

$$
\begin{aligned}
\mathbf{g}^{\mathrm{rot}} & =\mathbf{R}\left(-\theta_{\text {mix }}^{1},-\theta_{\text {mix }}^{2}\right) \mathbf{g R}\left(\theta_{\text {mix }}^{1}, \theta_{\text {mix }}^{2}\right) \\
& =\left[\begin{array}{cccc}
\left(\mathbf{g}_{\text {off }}^{\text {rot }}\right)_{11} & \left(\mathbf{g}_{\text {off }}^{\text {rot }}\right)_{12} & 0 & \left(\mathbf{g}_{\text {off }}^{\text {rot }}\right)_{14} \\
\left(\mathbf{g}_{\text {off }}^{\text {rot }}\right)_{21} & \left(\mathbf{g}_{\text {off }}^{\text {rot }}\right)_{22} & \left(\mathbf{g}_{\text {off }}^{\text {rot }}\right)_{23} & 0 \\
0 & \left(\mathbf{g}_{\text {off }}^{\text {rot }}\right)_{32} & \left(\mathbf{g}_{\text {off }}^{\text {rot }}\right)_{33} & \left(\mathbf{g}_{\text {off }}^{\text {rot }}\right)_{34} \\
\left(\mathbf{g}_{\text {off }}^{\text {rot }}\right)_{41} & 0 & \left(\mathbf{g}_{\text {off }}^{\text {rot }}\right)_{43} & \left(\mathbf{g}_{\text {off }}^{\text {rot }}\right)_{44}
\end{array}\right] .
\end{aligned}
$$

The diagonal elements are given by

$$
\begin{aligned}
\left(\mathbf{g}_{\text {diag }}^{\text {rot }}\right)_{11}= & -\frac{1}{2}\left(k_{\mathrm{AB}}+k_{\mathrm{BA}}+R_{S}^{0}(\mathrm{~A})+R_{S}^{0}(\mathrm{~B})\right. \\
& \left.+\sqrt{4 w^{2}+\left(k_{\mathrm{BA}}-k_{\mathrm{AB}}+R_{S}^{0}(\mathrm{~A})-R_{S}^{0}(\mathrm{~B})\right)^{2}}\right), \\
\left(\mathbf{g}_{\mathrm{diag}}^{\text {rot }}\right)_{22}= & -\frac{1}{2}\left(2 i \omega_{J}+k_{\mathrm{AB}}+k_{\mathrm{BA}}+R_{\Delta}^{0}(\mathrm{~A})+R_{\Delta}^{0}(\mathrm{~B})\right. \\
& \left.+\sqrt{4 w^{2}+\left(k_{\mathrm{BA}}-k_{\mathrm{AB}}+R_{\Delta}^{0}(\mathrm{~A})-R_{\Delta}^{0}(\mathrm{~B})\right)^{2}}\right), \\
\left(\mathbf{g}_{\mathrm{diag}}^{\text {rot }}\right)_{33}= & -\frac{1}{2}\left(k_{\mathrm{AB}}+k_{\mathrm{BA}}+R_{S}^{0}(\mathrm{~A})+R_{S}^{0}(\mathrm{~B})\right. \\
& \left.-\sqrt{4 w^{2}+\left(k_{\mathrm{BA}}-k_{\mathrm{AB}}+R_{S}^{0}(\mathrm{~A})-R_{S}^{0}(\mathrm{~B})\right)^{2}}\right), \\
\left(\mathbf{g}_{\mathrm{diag}}^{\text {rot }}\right)_{44}= & -\frac{1}{2}\left(2 i \omega_{J}+k_{\mathrm{AB}}+k_{\mathrm{BA}}+R_{\Delta}^{0}(\mathrm{~A})+R_{\Delta}^{0}(\mathrm{~B})\right. \\
& \left.-\sqrt{4 w^{2}+\left(k_{\mathrm{BA}}-k_{\mathrm{AB}}+R_{\Delta}^{0}(\mathrm{~A})-R_{\Delta}^{0}(\mathrm{~B})\right)^{2}}\right) .
\end{aligned}
$$

The non-zero off-diagonal elements are given by

$$
\begin{aligned}
& \left(\mathrm{g}_{\text {diag }}^{\text {rot }}\right)_{12}=\left(\mathbf{g}_{\text {diag }}^{\text {rot }}\right)_{21} \\
& =\frac{i}{\sqrt{3}}\left(\Delta \Omega^{\mathrm{A}} \mathrm{c}\left(\theta_{\text {mix }}^{1}\right) \mathrm{c}\left(\theta_{\text {mix }}^{2}\right)+\Delta \Omega^{\mathrm{B}} \mathrm{s}\left(\theta_{\text {mix }}^{1}\right) \mathrm{s}\left(\theta_{\text {mix }}^{2}\right)\right), \\
& \left(\mathrm{g}_{\text {diag }}^{\text {rot }}\right)_{14}=\left(\mathbf{g}_{\text {diag }}^{\text {rot }}\right)_{41} \\
& =\frac{i}{\sqrt{3}}\left(\Delta \Omega^{\mathrm{A}} \mathrm{c}\left(\theta_{\text {mix }}^{1}\right) \mathrm{s}\left(\theta_{\text {mix }}^{2}\right)-\Delta \Omega^{\mathrm{B}} \mathrm{s}\left(\theta_{\text {mix }}^{1}\right) \mathrm{c}\left(\theta_{\text {mix }}^{2}\right)\right), \\
& \left(\mathrm{g}_{\text {diag }}^{\text {rot }}\right)_{23}=\left(\mathrm{g}_{\text {diag }}^{\text {rot }}\right)_{32} \\
& =\frac{i}{\sqrt{3}}\left(\Delta \Omega^{\mathrm{A}} \mathrm{s}\left(\theta_{\text {mix }}^{1}\right) \mathrm{c}\left(\theta_{\text {mix }}^{2}\right)-\Delta \Omega^{\mathrm{B}} \mathrm{c}\left(\theta_{\text {mix }}^{1}\right) \mathrm{s}\left(\theta_{\text {mix }}^{2}\right)\right), \\
& \left(\mathrm{g}_{\text {diag }}^{\text {rot }}\right)_{34}=\left(\mathrm{g}_{\text {diag }}^{\text {rot }}\right)_{43} \\
& =\frac{i}{\sqrt{3}}\left(\Delta \Omega^{\mathrm{B}} \mathrm{c}\left(\theta_{\text {mix }}^{1}\right) \mathrm{c}\left(\theta_{\text {mix }}^{2}\right)+\Delta \Omega^{\mathrm{A}} \mathrm{s}\left(\theta_{\text {mix }}^{1}\right) \mathrm{s}\left(\theta_{\text {mix }}^{2}\right)\right),
\end{aligned}
$$

where we abbreviated $\mathrm{c}(x)=\cos (x)$ and $\mathrm{s}(x)=\sin (x)$.

For the case that $k_{\mathrm{BA}} \gg \omega_{J}$ the diagonal elements $\left(\mathbf{g}_{\text {off }}^{\text {rot }}\right)_{11}$ and $\left(\mathbf{g}_{\text {off }}^{\text {rot }}\right)_{22}$ are approximately equal, and as far as perturbation theory is concerned must be considered as quasi-degenerate. The quasi-degeneracy may be lifted via the basis transformation

$$
\mathbf{T}=\left[\begin{array}{cccc}
\frac{1}{\sqrt{2}} & \frac{1}{\sqrt{2}} & 0 & 0 \\
\frac{1}{\sqrt{2}} & -\frac{1}{\sqrt{2}} & 0 & 0 \\
0 & 0 & 1 & 0 \\
0 & 0 & 0 & 1
\end{array}\right]
$$

The starting point for our perturbative treatment thus becomes

$$
\mathbf{M}=\mathbf{T}^{-1} \mathbf{g}^{\mathrm{rot}} \mathbf{T}
$$

and an approximate expression for $R_{S}$ in configuration 
$\mathrm{B}$ is given by

$$
\begin{aligned}
R_{S}^{\mathrm{pert}} \simeq-\operatorname{Re}\left\{(\mathbf{M})_{33}\right\} & -\operatorname{Re}\left\{\sum_{j \in\{1,2,4\}}(\mathbf{M})_{3 j}(\mathbf{M})_{j 3} /\left((\mathbf{M})_{33}-(\mathbf{M})_{j j}\right)\right\} .
\end{aligned}
$$

After some algebraic manipulations this expression may be shown to reduce to equation 23 of the main text.

\section{REFERENCES}

${ }^{1}$ M. Carravetta, O. G. Johannessen, and M. H. Levitt, Physical Review Letters 92, 153003 (2004).

${ }^{2} \mathrm{M}$. Carravetta and M. H. Levitt, Journal of the American Chemical Society 126, 6228 (2004).

${ }^{3}$ M. Carravetta and M. H. Levitt, The Journal of Chemical Physics 122, 214505 (2005).

${ }^{4}$ G. Pileio, M. Carravetta, E. Hughes, and M. H. Levitt, Journal of the American Chemical Society 130, 12582 (2008).

${ }^{5}$ W. S. Warren, E. Jenista, R. T. Branca, and X. Chen, Science 323, 1711 (2009).

${ }^{6}$ G. Pileio and M. H. Levitt, The Journal of Chemical Physics 130, 214501 (2009)

${ }^{7}$ G. Pileio, M. Carravetta, and M. H. Levitt, Proceedings of the National Academy of Sciences 107, 17135 (2010).

${ }^{8}$ M. C. D. Tayler and M. H. Levitt, Physical Chemistry Chemical Physics 13, 5556 (2011).

${ }^{9}$ Y. Feng, R. M. Davis, and W. S. Warren, Nature Physics 8, 831 (2012), number: 11 Publisher: Nature Publishing Group.

${ }^{10}$ M. H. Levitt, Annu. Rev. Phys. Chem. 63, 89 (2012).

${ }^{11}$ G. Pileio, S. Bowen, C. Laustsen, M. C. D. Tayler, J. T. HillCousins, L. J. Brown, R. C. D. Brown, J. H. Ardenkjaer-Larsen, and M. H. Levitt, Journal of the American Chemical Society 135, 5084 (2013), publisher: American Chemical Society.

${ }^{12}$ G. Stevanato, S. S. Roy, J. Hill-Cousins, I. Kuprov, L. J. Brown, R. C. D. Brown, G. Pileio, and M. H. Levitt, Physical Chemistry Chemical Physics 17, 5913 (2015), publisher: The Royal Society of Chemistry.

${ }^{13} \mathrm{G}$. Stevanato, Long-lived states in multi-spin systems, phd, University of Southampton (2015).

${ }^{14}$ G. Stevanato, J. T. Hill-Cousins, P. Håkansson, S. S. Roy, L. J. Brown, R. C. D. Brown, G. Pileio, and M. H. Levitt, Angewandte Chemie International Edition 54, 3740 (2015).

${ }^{15} \mathrm{~S}$. J. Elliott, C. Bengs, L. J. Brown, J. T. Hill-Cousins, D. J. O'Leary, G. Pileio, and M. H. Levitt, The Journal of Chemical Physics 150, 064315 (2019).

${ }^{16}$ M. H. Levitt, Journal of Magnetic Resonance 306, 69 (2019).

${ }^{17}$ G. Pileio, ed., Long-lived Nuclear Spin Order: Theory and Applications, 1st ed. (Royal Society of Chemistry, S.l., 2020).

${ }^{18} \mathrm{M}$. H. Levitt, in Long-lived Nuclear Spin Order: Theory and Applications (Royal Society of Chemistry, 2020) 1st ed., p. 300.

${ }^{19}$ A. Moysiadi, F. Giustiniano, A. M. R. Hall, T. A. A. Cartlidge, L. J. Brown, and G. Pileio, Front Chem 9, 668044 (2021).

${ }^{20}$ S. Cavadini, J. Dittmer, S. Antonijevic, and G. Bodenhausen, Journal of the American Chemical Society 127, 15744 (2005).

${ }^{21}$ R. Sarkar, P. Ahuja, P. R. Vasos, and G. Bodenhausen, ChemPhysChem 9, 2414 (2008).

${ }^{22}$ S. Cavadini and P. R. Vasos, Concepts in Magnetic Resonance Part A 32A, 68 (2008).

${ }^{23}$ P. Ahuja, R. Sarkar, P. R. Vasos, and G. Bodenhausen, Journal of the American Chemical Society 131, 7498 (2009), publisher: American Chemical Society.

${ }^{24}$ R. Sarkar, P. Ahuja, P. R. Vasos, and G. Bodenhausen, Physical Review Letters 104, 053001 (2010).

${ }^{25}$ A. Bornet, P. Ahuja, R. Sarkar, L. Fernandes, S. Hadji, S. Y. Lee, A. Haririnia, D. Fushman, G. Bodenhausen, and P. R. Vasos, ChemPhysChem
12, $2729 \quad$ (2011), -eprint: https://chemistryeurope.onlinelibrary.wiley.com/doi/pdf/10.1002/cphc.201100365.

${ }^{26}$ Y. Zhang, P. C. Soon, A. Jerschow, and J. W. Canary, Angewandte Chemie International Edition 53, 3396 (2014).

${ }^{27}$ G. Pileio, J.-N. Dumez, I.-A. Pop, J. T. Hill-Cousins, and R. C. D. Brown, Journal of Magnetic Resonance 252, 130 (2015).

${ }^{28}$ G. Pileio and S. Ostrowska, Journal of Magnetic Resonance (San Diego, Calif.: 1997) 285, 1 (2017).

${ }^{29}$ M. C. Tourell, I.-A. Pop, L. J. Brown, R. C. D. Brown, and G. Pileio, Physical Chemistry Chemical Physics 20, 13705 (2018).

${ }^{30}$ N. Salvi, R. Buratto, A. Bornet, S. Ulzega, I. Rentero Rebollo, A. Angelini, C. Heinis, and G. Bodenhausen, Journal of the American Chemical Society 134, 11076 (2012).

${ }^{31}$ R. Buratto, A. Bornet, J. Milani, D. Mammoli, B. Vuichoud, N. Salvi, M. Singh, A. Laguerre, S. Passemard, S. Gerber-Lemaire, S. Jannin, and G. Bodenhausen, ChemMedChem 9, 2509 (2014), _eprint: https://chemistryeurope.onlinelibrary.wiley.com/doi/pdf/10.1002/cmdc.201402214.

${ }^{32}$ R. Buratto, D. Mammoli, E. Chiarparin, G. Williams, and G. Bodenhausen, Angewandte Chemie (International Ed. in English) 53, 11376 (2014).

${ }^{33}$ R. Buratto, D. Mammoli, E. Canet, and G. Bodenhausen, Journal of Medicinal Chemistry 59, 1960 (2016), publisher: American Chemical Society.

${ }^{34}$ N. Salvi, in Annual Reports on NMR Spectroscopy, Vol. 96, edited by G. A. Webb (Academic Press, 2019) pp. 1-33.

${ }^{35}$ P. R. Vasos, A. Comment, R. Sarkar, P. Ahuja, S. Jannin, J.-P. Ansermet, J. A. Konter, P. Hautle, B. v. d. Brandt, and G. Bodenhausen, Proceedings of the National Academy of Sciences 106, 18469 (2009).

${ }^{36}$ P. Ahuja, R. Sarkar, S. Jannin, P. R. Vasos, and G. Bodenhausen, Chemical Communications 46, 8192 (2010), publisher: Royal Society of Chemistry.

${ }^{37}$ Y. Feng, T. Theis, X. Liang, Q. Wang, P. Zhou, and W. S. Warren, Journal of the American Chemical Society 135, 9632 (2013), publisher: American Chemical Society.

${ }^{38}$ C. Laustsen, S. Bowen, M. S. Vinding, N. C. Nielsen, and J. H. Ardenkjaer-Larsen, Magnetic Resonance in Medicine 71, 921 (2014).

${ }^{39}$ S. S. Roy, P. J. Rayner, P. Norcott, G. G. R. Green, and S. B. Duckett, Physical Chemistry Chemical Physics 18, 24905 (2016).

${ }^{40}$ R. K. Ghosh, N. N. Kuzma, S. J. Kadlecek, and R. R. Rizi, Magnetic Resonance in Medicine 75, 1822 (2016), _eprint: https://onlinelibrary.wiley.com/doi/pdf/10.1002/mrm.25679.

${ }^{41}$ S. S. Roy, P. Norcott, P. J. Rayner, G. G. R. Green, and S. B. Duckett, Chemistry - A European Journal 23, 10496 (2017).

${ }^{42}$ A. S. Kiryutin, B. A. Rodin, A. V. Yurkovskaya, K. L. Ivanov, D. Kurzbach, S. Jannin, D. Guarin, D. Abergel, and G. Bodenhausen, Physical Chemistry Chemical Physics 21, 13696 (2019).

${ }^{43}$ K. F. Sheberstov, H.-M. Vieth, H. Zimmermann, B. A. Rodin, K. L. Ivanov, A. S. Kiryutin, and A. V. Yurkovskaya, Scientific Reports 9, 20161 (2019), number: 1 Publisher: Nature Publishing Group.

${ }^{44} \mathrm{~T}$. Jonischkeit, U. Bommerich, J. Stadler, K. Woelk, H. G. Niessen, and J. Bargon, The Journal of Chemical Physics 124, 201109 (2006), publisher: American Institute of Physics.

${ }^{45}$ D. Canet, S. Bouguet-Bonnet, C. Aroulanda, and F. Reineri, Journal of the American Chemical Society 129, 1445 (2007), publisher: American Chemical Society.

${ }^{46}$ T. Theis, P. Ganssle, G. Kervern, S. Knappe, J. Kitching, M. P. Ledbetter, D. Budker, and A. Pines, Nature Physics 7, 571 (2011), number: 7 Publisher: Nature Publishing Group.

${ }^{47}$ M. B. Franzoni, L. Buljubasich, H. W. Spiess, and K. Münnemann, "Long-Lived 1H Singlet Spin States Originating from Para-Hydrogen in Cs-Symmetric Molecules Stored for Minutes in High Magnetic Fields," (2012).

${ }^{48}$ L. Buljubasich, M. B. Franzoni, H. W. Spiess, and K. Münne- 
mann, Journal of Magnetic Resonance (San Diego, Calif.: 1997) 219, 33 (2012).

${ }^{49}$ V. V. Zhivonitko, K. V. Kovtunov, P. L. Chapovsky, and I. V. Koptyug, Angewandte Chemie 125, 13493 (2013), _eprint: https://onlinelibrary.wiley.com/doi/pdf/10.1002/ange.201307389.

${ }^{50} \mathrm{P}$. Türschmann, J. Colell, T. Theis, B. Blümich, and S. Appelt, Physical Chemistry Chemical Physics 16, 15411 (2014), publisher: Royal Society of Chemistry.

${ }^{51}$ D. Mammoli, B. Vuichoud, A. Bornet, J. Milani, J.-N. Dumez, S. Jannin, and G. Bodenhausen, The Journal of Physical Chemistry B 119, 4048 (2015), publisher: American Chemical Society.

${ }^{52}$ S. S. Roy, P. Norcott, P. J. Rayner, G. G. R. Green, and S. B. Duckett, Angewandte Chemie International Edition 55, $15642 \quad$ (2016), _eprint: https://onlinelibrary.wiley.com/doi/pdf/10.1002/anie.201609186.

${ }^{53}$ D. Graafen, M. B. Franzoni, L. M. Schreiber, H. W. Spiess, and K. Münnemann, Journal of Magnetic Resonance 262, 68 (2016).

${ }^{54}$ Y. Zhang, X. Duan, P. C. Soon, V. Sychrovský, J. W. Canary, and A. Jerschow, ChemPhysChem 17, 2967 (2016).

${ }^{55}$ Z. Zhou, J. Yu, J. F. P. Colell, R. Laasner, A. Logan, D. A. Barskiy, R. V. Shchepin, E. Y. Chekmenev, V. Blum, W. S. Warren, and T. Theis, The Journal of Physical Chemistry Letters 8, 3008 (2017), publisher: American Chemical Society.

${ }^{56}$ J. Eills, G. Stevanato, C. Bengs, S. Glöggler, S. J. Elliott, J. Alonso-Valdesueiro, G. Pileio, and M. H. Levitt, Journal of Magnetic Resonance 274, 163 (2017).

${ }^{57}$ B. Ripka, J. Eills, H. Kouřilová, M. Leutzsch, M. H. Levitt, and K. Münnemann, Chemical Communications 54, 12246 (2018).

${ }^{58}$ L. Dagys, B. Ripka, M. Leutzsch, G. A. I. Moustafa, J. Eills, J. F. P. Colell, and M. H. Levitt, Magnetic Resonance 1, 175 (2020), publisher: Copernicus GmbH.

${ }^{59}$ K. Miyanishi, N. Ichijo, M. Motoyama, A. Kagawa, M. Negoro, and M. Kitagawa, Quantum Science and Technology 5, 025004 (2020), publisher: IOP Publishing.

${ }^{60}$ E. Vinogradov and A. K. Grant, Journal of Magnetic Resonance 188, 176 (2007)

${ }^{61}$ A. K. Grant and E. Vinogradov, Journal of Magnetic Resonance 193, 177 (2008).

${ }^{62} \mathrm{~K}$. Gopalakrishnan and G. Bodenhausen, Journal of Magnetic Resonance 182, 254 (2006).

${ }^{63} \mathrm{G}$. Pileio and M. H. Levitt, Journal of Magnetic Resonance 187, 141 (2007)

${ }^{64}$ G. Pileio, Progress in Nuclear Magnetic Resonance Spectroscopy 56, 217 (2010)

${ }^{65}$ B. Meier, J.-N. Dumez, G. Stevanato, J. T. Hill-Cousins, S. S. Roy, P. Håkansson, S. Mamone, R. C. D. Brown, G. Pileio, and M. H. Levitt, Journal of the American Chemical Society 135 , 18746 (2013)

${ }^{66}$ J.-N. Dumez, P. Håkansson, S. Mamone, B. Meier, G. Stevanato, J. T. Hill-Cousins, S. S. Roy, R. C. D. Brown, G. Pileio, and M. H. Levitt, The Journal of Chemical Physics 142, 044506 (2015).

${ }^{67}$ S. J. Elliott, L. J. Brown, J.-N. Dumez, and M. H. Levitt, Physical Chemistry Chemical Physics 18, 17965 (2016).

${ }^{68}$ K. L. Ivanov, T. Kress, M. Baudin, D. Guarin, D. Abergel, G. Bodenhausen, and D. Kurzbach, The Journal of Chemical Physics 149, 054202 (2018)

${ }^{69}$ G. Pileio, The Journal of Chemical Physics 135, 174502 (2011), publisher: American Institute of Physics.

${ }^{70}$ C. Bengs, The Journal of Chemical Physics 152, 054106 (2020), publisher: American Institute of Physics.

${ }^{71}$ C. Bengs, The Journal of Chemical Physics 154, 244107 (2021), publisher: American Institute of Physics.

${ }^{72} \mathrm{~S}$. Cohen, J. R. Lacher, and J. D. Park, Journal of the American Chemical Society 81, 3480 (1959), publisher: American Chemical Society.

${ }^{73}$ J. D. Park, S. Cohen, and J. R. Lacher, Journal of the American Chemical Society 84, 2919 (1962), publisher: American Chemical Society.

${ }^{74} \mathrm{M}$. Ito and R. West, Journal of the American Chemical Society
85, 2580 (1963), publisher: American Chemical Society.

${ }^{75} \mathrm{R}$. West and D. L. Powell, Journal of the American Chemical Society 85, 2577 (1963), publisher: American Chemical Society.

${ }^{76} \mathrm{M}$. C. D. Tayler and M. H. Levitt, Journal of the American Chemical Society 135, 2120 (2013).

${ }^{77}$ P. E. Hansen, Progress in Nuclear Magnetic Resonance Spectroscopy 20, 207 (1988).

${ }^{78}$ L. M. Schwartz and L. O. Howard, J. Phys. Chem. 74, 4374 (1970).

${ }^{79}$ F. R. Wurm and H.-A. Klok, Chemical Society Reviews 42, 8220 (2013), publisher: Royal Society of Chemistry.

${ }^{80}$ I. V. Zhukov, A. S. Kiryutin, A. V. Yurkovskaya, Y. A. Grishin, H.-M. Vieth, and K. L. Ivanov, Physical Chemistry Chemical Physics 20, 12396 (2018), publisher: Royal Society of Chemistry.

${ }^{81}$ M. C. D. Tayler, in Long-lived Nuclear Spin Order (Royal Society of Chemistry, 2020) pp. 188-208.

${ }^{82}$ M. H. Levitt and R. Freeman, Journal of Magnetic Resonance (1969) 43, 502 (1981).

${ }^{83}$ S. Wimperis, Journal of Magnetic Resonance, Series A 109, 221 (1994).

${ }^{84}$ C. L. Perrin and J. D. Thoburn, J. Am. Chem. Soc. 114, 8559 (1992).

${ }^{85}$ T. L. Mega and R. L. Van Etten, J. Am. Chem. Soc. 115, 12056 (1993).

${ }^{86}$ C. Bengs, M. Sabba, A. Jerschow, and M. H. Levitt, Physical Chemistry Chemical Physics 22, 9703 (2020), publisher: The Royal Society of Chemistry.

${ }^{87}$ R. Kubo, Journal of Mathematical Physics 4, 174 (1963), publisher: American Institute of Physics.

${ }^{88}$ G. Binsch, Journal of the American Chemical Society 91, 1304 (1969), publisher: American Chemical Society.

${ }^{89}$ D. Abergel and A. G. Palmer, The Journal of Physical Chemistry B 109, 4837 (2005), publisher: American Chemical Society.

${ }^{90}$ R. R. Ernst, G. Bodenhausen, and A. Wokaun, Principles of Nuclear Magnetic Resonance in One and Two Dimensions, Vol. 1 (Clarendon Press, 1990).

${ }^{91}$ J. Cavanagh, W. J. Fairbrother, A. G. Palmer III Professor, N. J. Skelton, and M. Rance, Protein NMR Spectroscopy: Principles and Practice, 2nd ed. (Academic Press, Amsterdam ; Boston, 2006).

92 J. Kowalewski and L. Maler, Nuclear Spin Relaxation in Liquids: Theory, Experiments, and Applications, Second Edition, 2nd ed. (CRC Press, Boca Raton, 2017).

${ }^{93} \mathrm{C}$. Bengs and M. H. Levitt, Journal of Magnetic Resonance , 106645 (2019).

${ }^{94}$ L. R. Martins, M. C. C. Ribeiro, and M. S. Skaf, The Journal of Physical Chemistry B 106, 5492 (2002), publisher: American Chemical Society.

${ }^{95} \mathrm{Z}$. Luz and S. Meiboom, Journal of the American Chemical Society 85, 3923 (1963), publisher: American Chemical Society.

${ }^{96} \mathrm{~J}$. Wallerstein, U. Weininger, M. A. I. Khan, S. Linse, and M. Akke, J. Am. Chem. Soc. 137, 3093 (2015).

${ }^{97}$ U. Haupts, S. Maiti, P. Schwille, and W. W. Webb, Proceedings of the National Academy of Sciences 95, 13573 (1998), publisher: National Academy of Sciences Section: Biological Sciences.

${ }^{98}$ B. Zelent, J. M. Vanderkooi, R. G. Coleman, I. Gryczynski, and Z. Gryczynski, Biophysical Journal 91, 3864 (2006).

${ }^{99}$ D. T. Gillespie, Physica A: Statistical Mechanics and its Applications 188, 404 (1992).

${ }^{100}$ D. T. Gillespie, American Journal of Physics 64, 225 (1996), publisher: American Association of Physics Teachers.

${ }^{101}$ N. G. Van Kampen, Stochastic Processes in Physics and Chemistry (Elsevier, 2007).

${ }^{102}$ T. I. Igumenova, U. Brath, M. Akke, and A. G. Palmer, Journal of the American Chemical Society 129, 13396 (2007), publisher: American Chemical Society.

${ }^{103}$ P. Vallurupalli, G. Bouvignies, and L. E. Kay, Journal of the American Chemical Society 134, 8148 (2012), publisher: Amer- 
ican Chemical Society.

${ }^{104}$ J. R. Bothe, Z. W. Stein, and H. M. Al-Hashimi, Journal of Magnetic Resonance 244, 18 (2014).

${ }^{105}$ Y. Zhou and D. Yang, Journal of Magnetic Resonance 249, 118 (2014).

106 A. G. Palmer, Journal of Magnetic Resonance (San Diego, Calif.: 1997) 241, 3 (2014).

${ }^{107}$ A. G. Palmer and H. Koss, in Methods in Enzymology, Biological NMR Part B, Vol. 615, edited by A. J. Wand (Academic Press, 2019) pp. 177-236.

${ }^{108}$ T. R. Alderson and L. E. Kay, Current Opinion in Structural Biology Folding and Binding Proteins, 60, 39 (2020).

${ }^{109}$ R. W. Adams, J. A. Aguilar, K. D. Atkinson, M. J. Cowley, P. I. P. Elliott, S. B. Duckett, G. G. R. Green, I. G. Khazal, J. López-Serrano, and D. C. Williamson, Science 323, 1708 (2009).

${ }^{110}$ T. Theis, M. L. Truong, A. M. Coffey, R. V. Shchepin, K. W. Waddell, F. Shi, B. M. Goodson, W. S. Warren, and E. Y. Chekmenev, J. Am. Chem. Soc. 137, 1404 (2015).

${ }^{111}$ D. A. Barskiy, S. Knecht, A. V. Yurkovskaya, and K. L. Ivanov, Progress in Nuclear Magnetic Resonance Spectroscopy 114115, 33 (2019).

${ }_{112}$ R. G. Lawler, Accounts Chem. Res. 5, 25 (1972).

${ }^{113}$ P. J. Hore and R. W. Broadhurst, Prog. Nucl. Magn. Reson. Spectrosc. 25, 345 (1993).

${ }^{114}$ G. Buntkowsky, B. Walaszek, A. Adamczyk, Y. Xu, H.-H. Limbach, and B. Chaudret, Phys. Chem. Chem. Phys. 8, 1929
(2006).

${ }^{115}$ S. R. Logan, Trans. Faraday Soc. 63, 3004 (1967).

${ }^{116}$ R. Radeglia, Solid State Nuclear Magnetic Resonance 4, 317 (1995).

${ }^{117}$ R. P. Young, C. R. Lewis, C. Yang, L. Wang, J. K. Harper, and L. J. Mueller, Magn. Reson. Chem. 57, 211 (2019).

${ }^{118}$ C. Bengs and M. H. Levitt, Magnetic Resonance in Chemistry 56, 374 (2018).

${ }^{119}$ M. J. Frisch, G. W. Trucks, H. B. Schlegel, G. E. Scuseria, M. A. Robb, J. R. Cheeseman, G. Scalmani, V. Barone, G. A. Petersson, H. Nakatsuji, X. Li, M. Caricato, A. V. Marenich, J. Bloino, B. G. Janesko, R. Gomperts, B. Mennucci, H. P. Hratchian, J. V. Ortiz, A. F. Izmaylov, J. L. Sonnenberg, Williams, F. Ding, F. Lipparini, F. Egidi, J. Goings, B. Peng, A. Petrone, T. Henderson, D. Ranasinghe, V. G. Zakrzewski, J. Gao, N. Rega, G. Zheng, W. Liang, M. Hada, M. Ehara, K. Toyota, R. Fukuda, J. Hasegawa, M. Ishida, T. Nakajima, Y. Honda, O. Kitao, H. Nakai, T. Vreven, K. Throssell, J. A. Montgomery Jr., J. E. Peralta, F. Ogliaro, M. J. Bearpark, J. J. Heyd, E. N. Brothers, K. N. Kudin, V. N. Staroverov, T. A. Keith, R. Kobayashi, J. Normand, K. Raghavachari, A. P. Rendell, J. C. Burant, S. S. Iyengar, J. Tomasi, M. Cossi, J. M. Millam, M. Klene, C. Adamo, R. Cammi, J. W. Ochterski, R. L. Martin, K. Morokuma, O. Farkas, J. B. Foresman, and D. J. Fox, "Gaussian 16 Rev. C.01," (2016).

${ }^{120}$ R. J. Wittebort and A. Szabo, The Journal of Chemical Physics 69, 1722 (1978), publisher: American Institute of Physics. 